\title{
A sunlight self-healable fibrous flexible pressure sensor based on electrically conductive composite wool yarns
}

\author{
Y. X. Song, W. M. Xu, M. Z. Rong, M. Q. Zhang* \\ Key Laboratory for Polymeric Composite and Functional Materials of Ministry of Education, GD HPPC Lab, School \\ of Chemistry, Sun Yat-sen University, 510275 Guangzhou, P. R. China
}

Received 20 March 2020; accepted in revised form 7 May 2020

\begin{abstract}
The present work proposed a simple approach for producing a fibrous flexible resistive-type pressure sensor from wool. By dip-coating polyurethane (PU) and silver nanowires (AgNWs) onto wool yarn, the latter was turned into a coaxial cable-like structure with the electrically conductive AgNWs/PU composite sheath for the sensing functionality and the wool core for providing a pliable substrate of the sensor. Afterward, the overlapping region of two strands of orthogonally stacked conductive composite yarns was employed to act as the pressure sensor. The experimental results indicated that conductivity and durability of the conductive yarn and sensitivity of the pressure sensor are closely correlated and can be effectively tuned through changing the amounts of PU and AgNWs. The surface fine structure of the composite yarns is a key factor determining responsive behavior. As a result of optimization, the pressure sensor has acquired high sensitivity and longterm working stability. Moreover, the homemade PU imparted the sensor with sunlight triggered self-healability of microcracks. It is anticipated that the pressure sensor possesses promising application potential in a variety of areas, including wearable electronics and e-textiles.
\end{abstract}

Keywords: biopolymers, biocomposites, fibrous pressure sensor, wool, sunlight self-healing

\section{Introduction}

Flexible pressure sensors, a kind of transducers that convert the changes in external force into electrical signals, have attracted increasing research interests for their potential applications in wearable electronics $[1,2]$, soft robotics [3, 4], health monitoring [5, 6] and human-machine interfacing [7-9]. To date, the reported flexible pressure sensors are typically based on piezoresistive [10-12], piezoelectric [13, 14], capacitive $[15,16]$, and triboelectric [17-19] sensing mechanisms. Comparatively, piezoresistive pressure sensors, which depend on the change in resistance of a material when deformed, are more popular owing to their superior sensitivity, simplicity of device fabrication, and low energy consumption in operation. As for the application forms of flexible pressure sensors, the research efforts so far [20-24] in this aspect are mainly focused on 2D film-based volumetric sensors, which usually employ polymeric materials as substrates. Nevertheless, this type of pressure sensors still has critical disadvantages such as complicated fabrication processes, high-temperature treatment, and inapplicability to irregular and arbitrary surfaces, which limit the promising utilization.

Meanwhile, 1D fiber-based pressure sensors are developed in accordance with the trend of miniaturization of electric devices. As they can be woven into arbitrary shapes of fabrics and clothes, which are more breathable and comfortable than the polymeric substrates employed by the 2D film-based versions, their application scope, especially in wearable electronic textiles (e-textiles), is greatly broadened.

To achieve a high-performance flexible 1D fiberbased pressure sensor, highly electro-conductive 
fibers with low power consumption are fundamentally essential. Flexible conductive materials like conductive polymers $[25,26]$, metallic nanoparticles/ nanowires [27-29], and carbon materials (including carbon nanotubes [30-32] and graphene [33, 34]) have been investigated. For example, a highly stretchable coaxial fiber conductor with a conductivity of $461 \mathrm{~S} / \mathrm{cm}$ was prepared, in which conductive polymer ribbons were embedded inside thermoplastic elastomer channels [25]. A sheath-core conductive fiber created by wrapping carbon nanotube sheets oriented in the fiber direction had a conductivity of $360 \mathrm{~S} / \mathrm{cm}$ [30]. Cheng et al. [34] developed a graphene-based fiber with a conductivity of $0.136 \mathrm{~S} / \mathrm{m}$. It is worth noting that, however, poorer stability and durability of conductive polymers, the risk of pulmonary toxicity of carbon nanotubes, and lower conductivity of graphene-based fibers obstruct their applications. In this context, silver nanowires (AgNWs), characterized by the features of mechanically robust yet flexible, excellent electric conductivity, antimicrobial properties, and good environmentally durability, become rather competitive.

Besides, sensitivity is a key metric to quantify the quality of sensors. As reflected by the works about 2D film-based pressure sensors, the construction of appropriate microstructures may help to realize high sensitivity. Pan et al. [35] presented an ultra-sensitive resistive pressure sensor based on a hollowsphere micro-structured conducting polymer thin film. Bae et al. [36] reported a highly sensitive resistive pressure sensor with a linear response to the applied pressure by making use of a hierarchical graphene array as an electrode material and polydimethylsiloxane (PDMS) as the flexible substrate. In addition, a vertically aligned carbon nanotube forest embedded in the PDMS matrix with irregular surface morphology proved to be rather sensitive for resistive pressure sensing [37]. Although these microstructures are very useful for constructing fragile conduction paths, the fabrication processes require complicated synthesis or rigorous conditions and are hard to be applied for the 1D-based fiber pressure sensors.

In this study, we propose a facile and low-cost way to produce pressure sensor with low power consumption based on conductive fibrous material (Figure 1). Firstly, we plan to employ lightweight wool yarns as the flexible substrate, of which the surfaces with wavelike microstructure would easily morph and

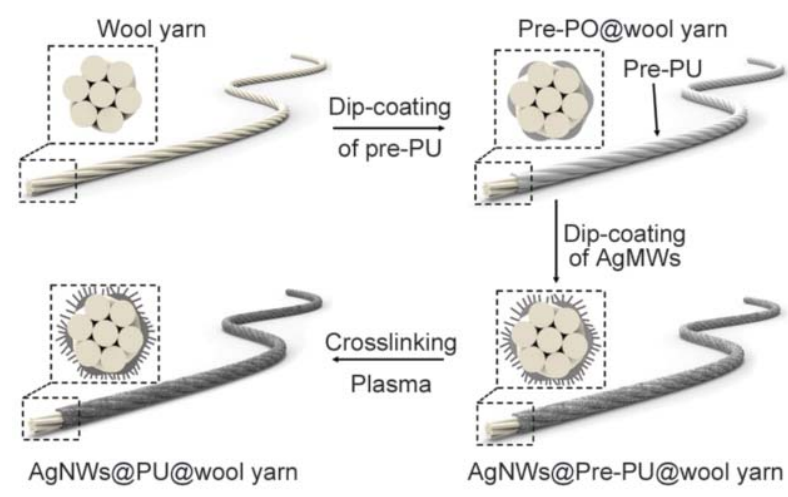

Figure 1. Schematic illustration of the fabrication of the wool-based conductive composite yarn.

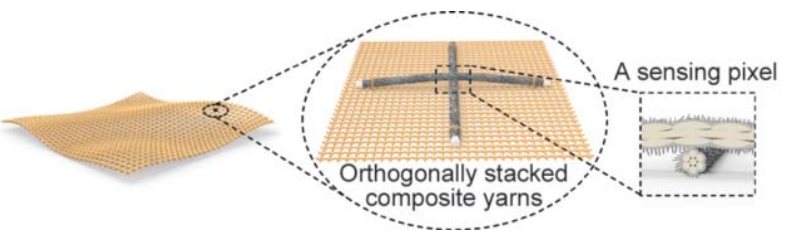

Figure 2. The pressure sensor fabricated by orthogonally stacking the conductiveAgNWs@PU@wool yarns.

hence beneficial to the improvement of pressure sensitivity of the sensor, not to mention the favorite biocompatibility and biodegradability associated with animal fibers. Secondly, electrical conductivity is imparted to the wool yarns by introducing a crosslinked polyurethane (PU) made in our lab [38] as an adhesive to glue AgNWs networks and the yarns together. The PU not only possesses optical transparency and yellowing resistance but also can be self-healed under sunshine through the reversible exchange reaction of the included disulfide bonds in the main chains. The latter functionality would recover the damages generated during the service of the sensor so that its working life can be extended. Thirdly, two strands of the conductive wool-based composite yarns are orthogonally stacked together, and the overlapping zone serves as the pressure sensor (Figure 2). In this case, the electrical resistance generated by the point contacted AgNWs networks would be rather sensitive to the applied stress.

Hereinafter, the feasibility of the above material design is verified, and the key influential factors are discussed as a function of the properties of the sensor.

\section{Experimental}

\subsection{Materials and reagents}

Raw merino fibers (diameter $\approx 35-45 \mu \mathrm{m}$ ) were purchased from Shanghai Yuanheng Wool Products Co., China. Before use, the fibers were soaked in a solution of $0.5 \mathrm{wt} \%$ sodium laurylsulfate at a wool-to-solution 
weight ratio of 1:60 at room temperature for $30 \mathrm{~min}$ [39]. Next, the wool fibers were washed in distilled water and then dried in air.

Isophorone diisocyanate (IPDI, 99\%), polyethylene glycol (PEG400, $\left.M_{\mathrm{w}}=400\right)$, tris(2-hydroxyethyl) amine (TEA, 99\%), and ditin butyl dilaurate (95\%) were supplied by Shanghai Aladdin Bio-Chem Technology Co., China. 2,2'-Hydroxy ethyldisulfide (HEDS, technical grade) was provided by Shanghai Sigma-Aldrich LLC., China. All the reagents were dried before use.

Silver nitrate $\left(\mathrm{AgNO}_{3}, 99.8 \%\right)$ and polyvinylpyrrolidone $\left(\mathrm{k}-90, M_{\mathrm{w}}=130000\right)$ were purchased from Acros. Ethylene glycol (AR), concentrated hydrochloric acid (AR), ethyl alcohol (AR), and dichloromethane (HPLC) were supplied by Guangzhou Chemical Reagent Factory, China. All the reagents and solvents were used without further treatment.

\subsection{Synthesis}

\section{Synthesis of PU}

The sunlight-driven healable polyurethane was synthesized according to the approach described elsewhere [38]. Typically, dried IPDI (0.022 mol), HEDS $(0.01 \mathrm{~mol})$, and PEG400 $(0.01 \mathrm{~mol})$ were added to $100 \mathrm{ml}$ dichloromethane. The mixture with one drop of ditin butyl dilaurate was stirred at $60^{\circ} \mathrm{C}$ for $12 \mathrm{~h}$ in an argon atmosphere. Then, TEA (0.0013 mol) was incorporated into the solution, and the system was kept stirring for $6 \mathrm{~h}$ to obtain the pre-crosslinked polyurethane solution. At last, the solution was cooled down and diluted to $25 \mathrm{wt} \%$ with dichloromethane for the subsequent fabrication of the target pressure sensor.

\section{Synthesis of AgNWs}

AgNWs were synthesized by using the one-step polyol method [40]. Typically, silver nitrate $\left(2.35 \cdot 10^{-2} \mathrm{~mol}\right)$ and polyvinylpyrrolidone (PVP, $3.6 \cdot 10^{-2} \mathrm{~mol}$ ) were respectively dissolved in $200 \mathrm{ml}$ ethylene glycol and then transferred into a $500 \mathrm{ml}$ polyvinylidene fluoride (PVDF) digestion tank. $50 \mathrm{ml}$ hydrochloric acid in ethylene glycol solution with a molarity of $5 \cdot 10^{-3} \mathrm{~mol} / 1$ was poured into the digestion tank and well mixed with the others. The tank was sealed and heated at $140^{\circ} \mathrm{C}$ for $15 \mathrm{~h}$. Afterwards, the solution was quenched in a room temperature water bath to terminate the reaction. When the AgNWs solution was cooled, it was centrifuged at $5000 \mathrm{rpm}$ for $10 \mathrm{~min}$ and washed three times with ethanol to remove the excess PVP and ethylene glycol. Lastly, AgNWs were diluted in ethanol to obtain a dispersion solution of $6 \mathrm{mg} / \mathrm{ml}$. The length and diameter of the produced AgNWs were 100-150 mm and $100-150 \mathrm{~nm}$, respectively.

\section{Fabrication of the pressure sensor}

The cleaned wool fibers were first spun into yarns with a diameter of about $1 \mathrm{~mm}$ as the flexible substrate of the pressure sensor. Next, the wool yarns were dip-coated with $25 \mathrm{wt} \%$ pre-crosslinked polyurethane in dichloromethane for different times to obtain Pre-PU@wool yarns. When the solvent was evaporated at $25^{\circ} \mathrm{C}$ for $12 \mathrm{~h}$, the Pre-PU@wool yarns were dip-coated with $\mathrm{AgNWs}$ dispersion solution of $6 \mathrm{mg} / \mathrm{ml}$ at $60^{\circ} \mathrm{C}$ for different times. Next, the composite yarns (AgNWs@Pre-PU@wool yarns) were heated at $60^{\circ} \mathrm{C}$ for an additional $12 \mathrm{~h}$ to completely crosslink the PU. Afterward, the sample surface was treated by argon plasma for $20 \mathrm{~min}$ at room temperature using a PT-2S plasma surface treatment apparatus $(150 \mathrm{~W}$ and $40 \mathrm{kHz}$, Sanhe Boda Mechanical and Electrical Technology Co., Ltd., Shenzhen, China), offeringAgNWs@PU@wool composite yarns for the subsequent tests. The plasma treatment removed the insulating residual PVP on the surface of AgNWs and welded the junctions of AgNWs (Figure 3), which increased conductivity of the AgNWs and mechanical robustness of the networked AgNWs [40].

For the convenience of discussion, the times of dipcoating of the solutions of pre-crosslinked PU (m) and AgNWs dispersion (n) are included in the code names of the conductive composite yarns as AgNWs(m)@PU(n)@wool.

AgNWs(100)@PU(2)@wool, for example, represents the yarns dip-coated by the pre-crosslinked PU solution for two times and AgNWs dispersion solution for 100 times.

Two strands of the above conductive composite yarns were perpendicularly sewed onto a piece of soft and thin cotton fabric. The point-to-point resistive sensing pixel formed at the crisscross location of the yarns serves as the pressure sensor.

\subsection{Characterization}

To study the resistance change of single conductive yarn caused by cyclic bending deformation and that of the pressure sensor caused by cyclic compression, a CMT 6000 universal tester (SANS, Shenzhen, China), combined with a $\mathrm{CH} 660 \mathrm{C}$ electrochemical 


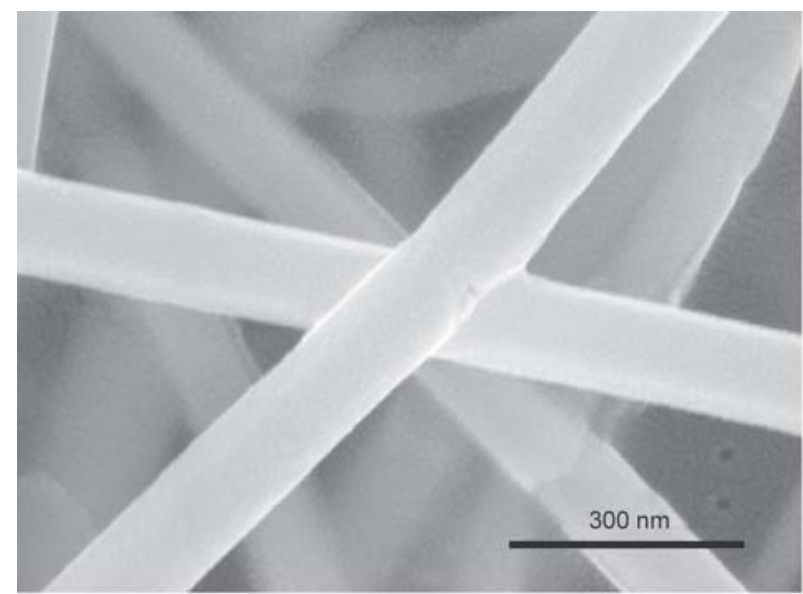

a)

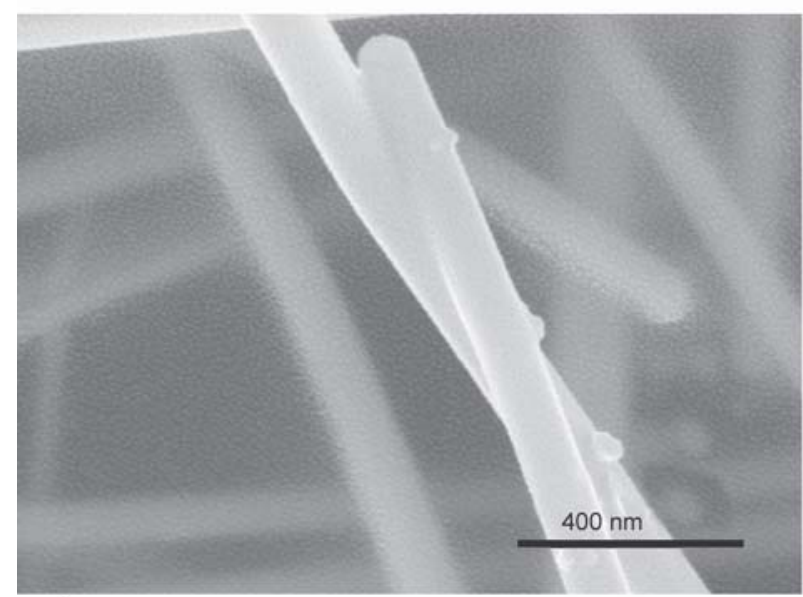

c)

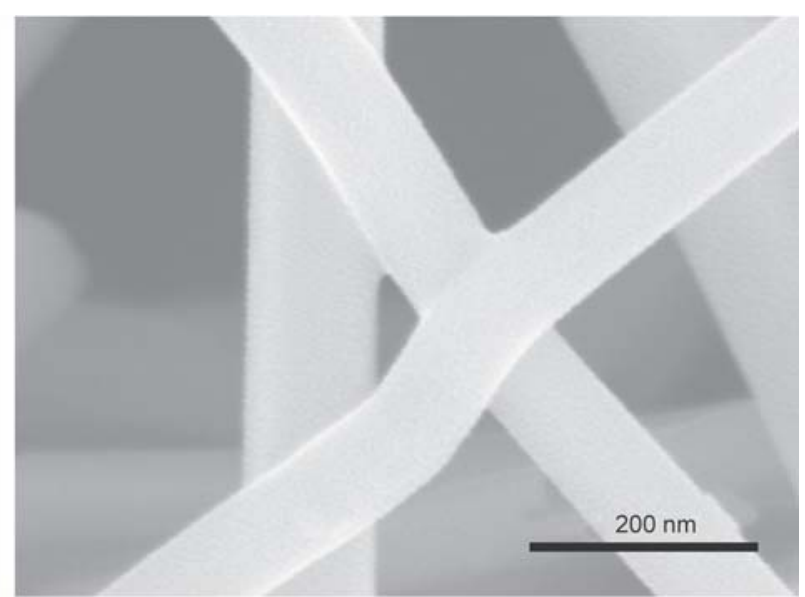

b)

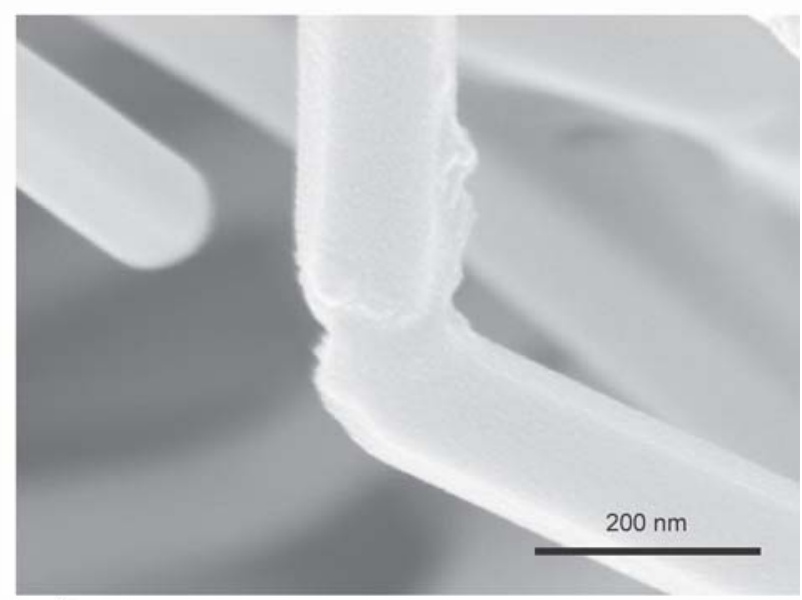

d)

Figure 3. SEM photos showing the welded AgNWs after plasma treatment (a-d).

work station (Chenhua Instrument Co., Ltd., Shanghai, China), was employed. The change in electrical resistance is defined as $\Delta R / R_{0}$, where $R_{0}$ denotes the initial resistance, and $\Delta R$ is the difference between the measured resistance under the applied pressure and the initial resistance.

For the cyclic bending experiments, the bending angle ranged between 10 and $180^{\circ}$, and the frequency was fixed at $0.5 \mathrm{~Hz}$ (Figure $4 \mathrm{a}$ ). With respect to the cyclic compression tests, the force gauge of the universal tester was pressed onto a piece of a squareshaped glass slide $(10 \mathrm{~mm} \times 10 \mathrm{~mm})$ to evenly apply the normal pressure onto the center of the sensing pixel (Figure 4b). During the measurements, the specimens were compressed to a certain force, $F$, from the initial displacement under a crosshead speed of $3 \mathrm{~mm} / \mathrm{min}$ at $25^{\circ} \mathrm{C}$ and then released to the initial displacement under the same crosshead speed. The same cycle was repeated multiple times. The apparent pressure, $P$, was calculated by $P=F / A$, where $A$ is the area of the glass slide (i.e. $100 \mathrm{~mm}^{2}$ ). The sensitivity of the pressure sensors was estimated from the absolute value of the slope of relative resistance variation versus pressure.

We also measured the resistance variations of a single strand of conductive composite yarn with the applied pressure, but the results were far lower than those detected from the orthogonally stacked yarns. It was thus concluded that the single composite yarn alone is not a suitable form of pressure sensor application. The morphological observation was conducted by a Model S-4800 (Hitachi, Tokyo, Japan) field emission scanning electron microscope (SEM).

For examination of the capability of autonomous restoring the electrical conductivity of the AgNWs@PU@wool yarn, the specimen was first immersed into liquid nitrogen and tapped with a hammer to create micro-cracks on the AgNWs@PU composite. Then, the damaged specimen was healed under sunlight from 9:00 am to 4:00 pm for two days in September in Guangzhou, China (intensity of solar radiation $=60-90 \mathrm{~mW} / \mathrm{cm}^{2}$ ). The electrical healing efficiency was calculated from the ratio of the difference in resistance between the damaged and 


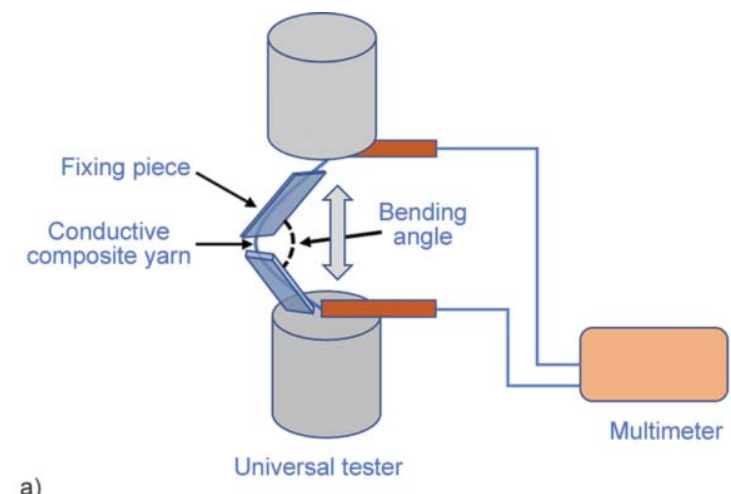

a)

Figure 4. Configurations for testing repeated resistance variations in response to (a) cyclic bending and (b) cyclic compressive pressure.

healed specimen to that between the damaged and virgin specimen.

For exploring the e-textile application of the pressure sensor, electrical responses of the pressure sensors were recorded in real time using Keithley 4200-SC digital meter (Keithley Instruments, Inc., Cleveland, USA) with a time interval of $60 \mathrm{~ms}$.

\section{Results and discussion}

In the beginning, the influences of manufacturing processes and formulation on electrical resistance and performance stability of the wool-based conductive composite yarns are discussed for optimizing the processing, which determines the quality of the ultimate sensor. As illustrated in Figure 1, two main steps are involved in the production of AgNWs@PU@wool yarns: dip-coating of the solutions of (i) pre-crosslinked PU and (ii) AgNWs dispersion. The effect of the first step can be seen from the SEM photos in Figure 5 and Figure 6. It shows that the wool yarns are tightly cohered, forming compact integrity after the dip-coating of the pre-crosslinked PU solution. With a rise in the dip-coating times, the pre-crosslinked PU layer attached to the wool yarn gradually becomes thicker as more PU is introduced. Moreover, the surface fine texture of wool fibers becomes nearly invisible when the dip-coating is carried out three times or more. Since (i) the uneven surface profiles are conducive to the production of pressure sensor with high sensitivity as analyzed in the Introduction, and (ii) the amount of the precrosslinked PU should not be too few for capturing AgNWs, we deduce that the PU@wool yarns made by dip-coating of the pre-crosslinked PU solution for two times are the optimal ones for the following experiments.

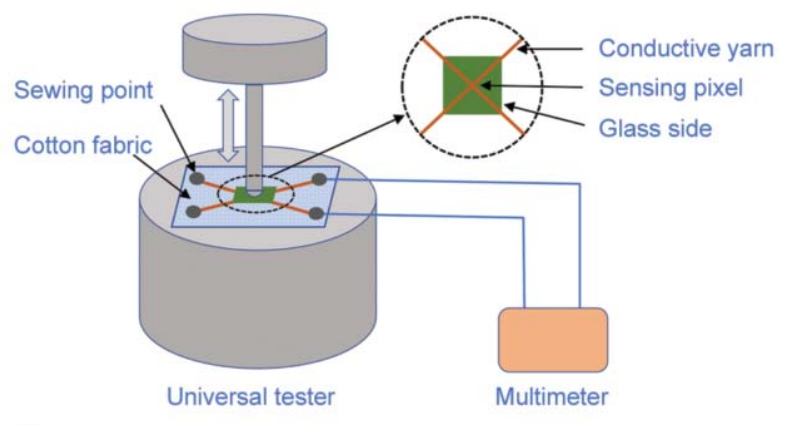

b)
As for the second step, the PU@wool yarns are dipcoated with AgNWs dispersion solution to acquire electrical conductivity. Figure 7 displays that the resistance of the resultantAgNWs@PU@wool yarns decreases with increasing the dip-coating times. It tends to be stabilized at $0.95 \Omega / \mathrm{cm}$ as the AgNWs are dip-coated for 100 times, which is comparable to the value of a commercialized conductive thread $(1 \Omega / \mathrm{cm})$ [8]. The reason accounting for the declining trend exhibited in Figure 7 lies in the fact that more AgNWs adhere to the PU@wool yarn (Figure 8), the more dense and compact conduction networks are established. When the interstitial gaps among the existing AgNWs are filled with sufficient amount of additional AgNWs, the stability of the conduction networks can be ensured in addition to the high conductivity, but the resistance change in response to the applied stress, i.e. sensitivity of the proposed pressure sensor, would not be high enough as the relative movement of the AgNWs driven by the applied force has to be restricted at the same time. Evidently, the stability and sensitivity of the conductive composite yarns are conflicting, and we have to find a balance between the two factors.

Figure 9a further quantifies the combined influences of the dip-coating times of pre-crosslinked PU and AgNWs solutions on the conductivity of the composite yarns. Compared to the times of dip-coating of AgNWs solutions, the times of dip-coating of precrosslinked PU (i.e. the PU content) do not lead to a significant difference in resistance within the range of interests. This is understandable as the conductivity of the composite yarn is determined by the dosage of the conducting fillers, i.e. AgNWs, after all.

Besides conductivity, the durability of the conductive composite yarns should also be characterized. 


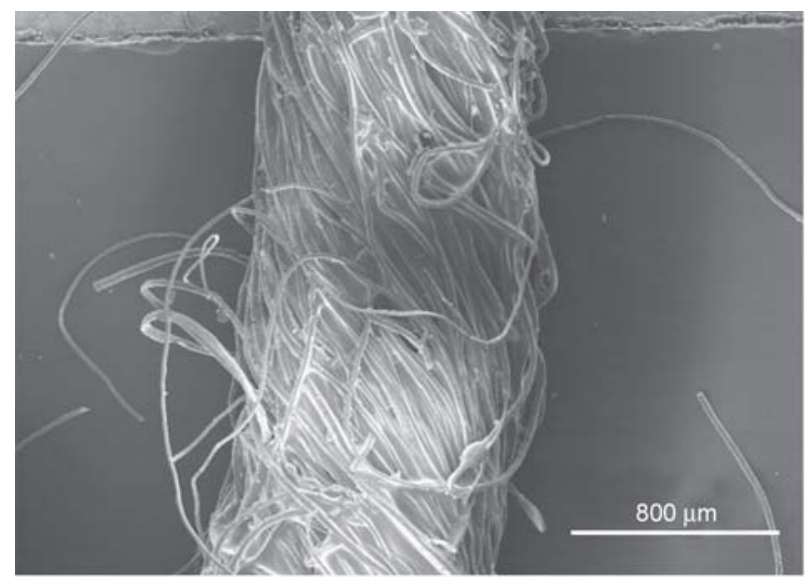

a)

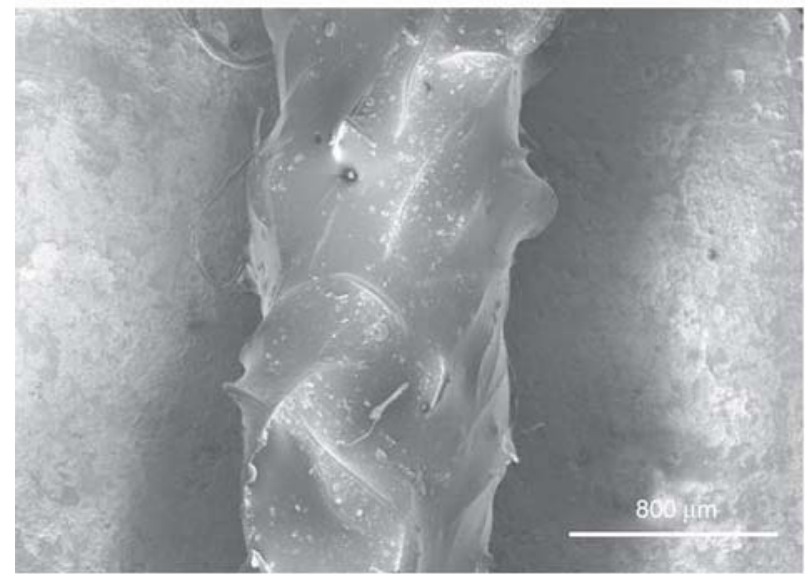

c)

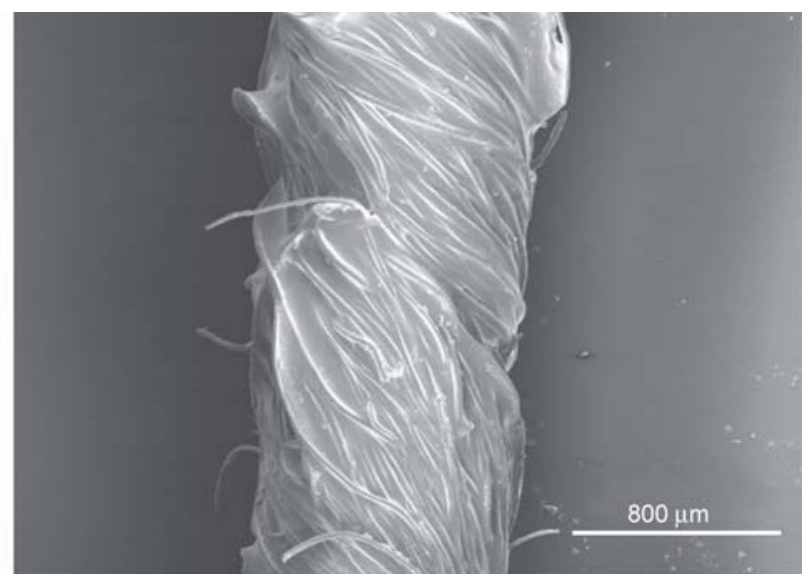

b)

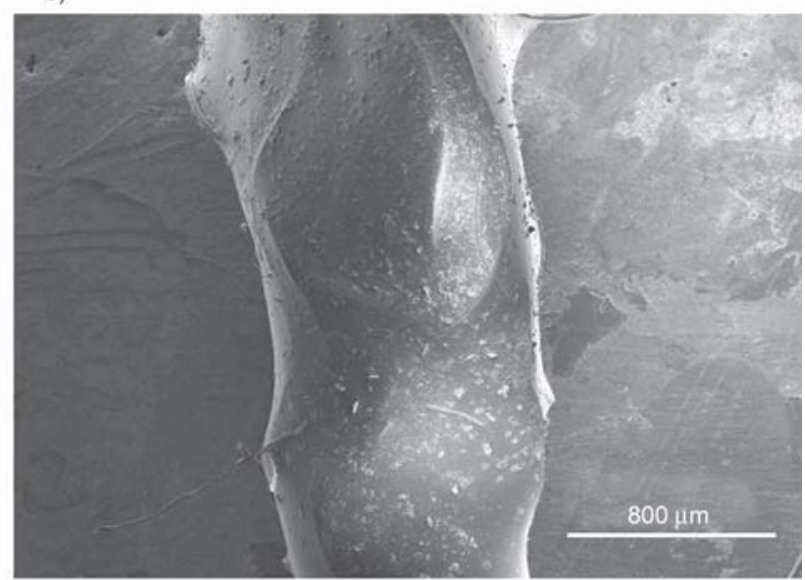

d)

Figure 5. SEM photos of the side views of the wool yarns dip-coated with the pre-crosslinked PU solution for (a) once, (b) twice, (c) thrice, and (d) four times.

Figure $9 \mathrm{~b}$ depicts the variations in resistance of the conductive composite yarns subjected to repeated bending deformation. The data indicate that the electrical resistance of the AgNWs(70)@PU(1)@wool yarn, which is respectively dip-coated with the precrosslinked PU for only once and AgNWs solution for 70 times, remarkably increases with the bending cycles. The insufficient interfacial adhesion between AgNWs and wool (Figure 5a) must account for the instability, as the increased integrity of the AgNWs networks by plasma welding doesn't help in this case. When the amount of PU is high enough to hold the AgNWs, the variation in resistance induced by cyclic bending is marginal (refer to

AgNWs(100)@PU(2)@wool, AgNWs(70)@PU(2)@wool, AgNWs(70)@PU(4)@wool and AgNWs(40)@PU(2)@wool), in spite of the fact that the content of AgNWs of certain composite yarn (i.e.AgNWs(40)@PU(2)@wool) is far lower than the equilibrium value (Figure 7).
The results reveal the importance of PU content for the robustness of the conductive composite yarns. By using the above preparedAgNWs@PU@wool yarns, the pressure sensors are assembled as planned (Figure 2). The plots in Figure 10a show the relative resistance variations of the sensors along with the applied pressure and the corresponding sensitivity as well. It is clear that all the samples exhibit reduction in resistance when the pressure increases owing to the increase of conduction paths. For the sensors composed of AgNWs@PU@wool yarns containing different concentrations of AgNWs at a constant fraction of PU, their sensitivities decrease with increasing the dip-coating times of AgNWs dispersion solution. The pressure sensor made by the AgNWs@PU@wool yarns dip-coated with AgNWs dispersion solution for 40 times shows the highest sensitivities, while the sensor from the conductive yarns dip-coated with AgNWs dispersion for 100 times has the lowest sensitivities. On the whole, the sensitivities of our wool derived pressure sensors are comparable with those 


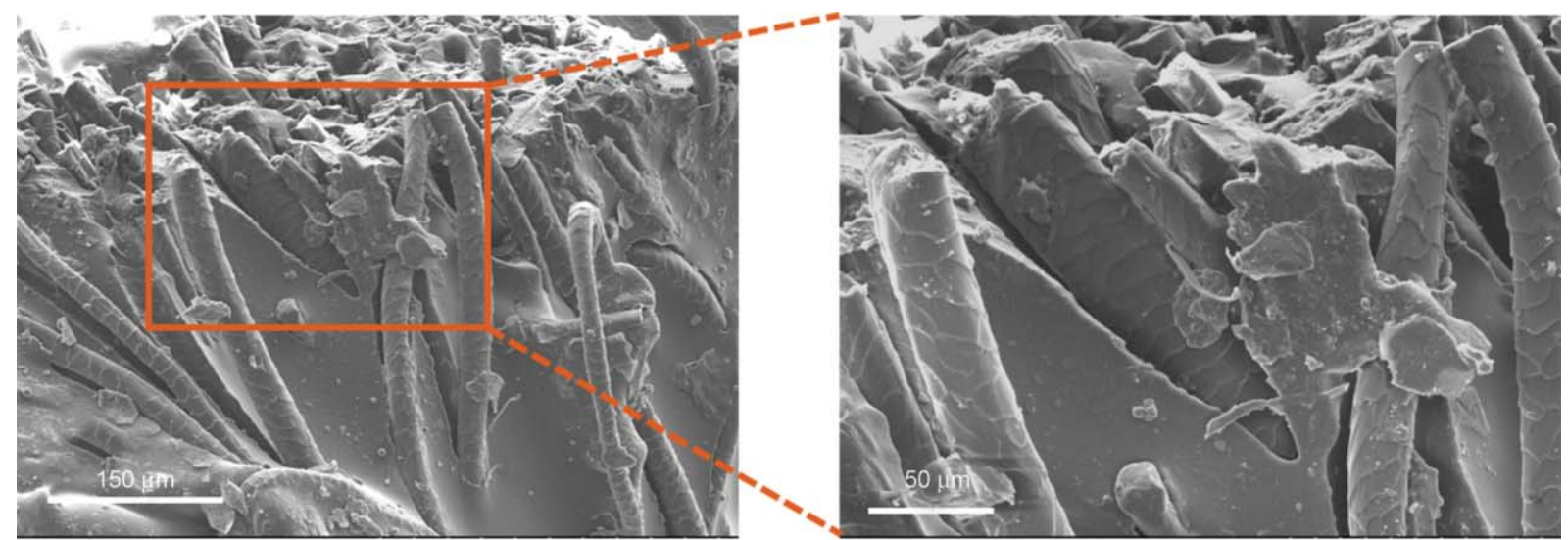

a)
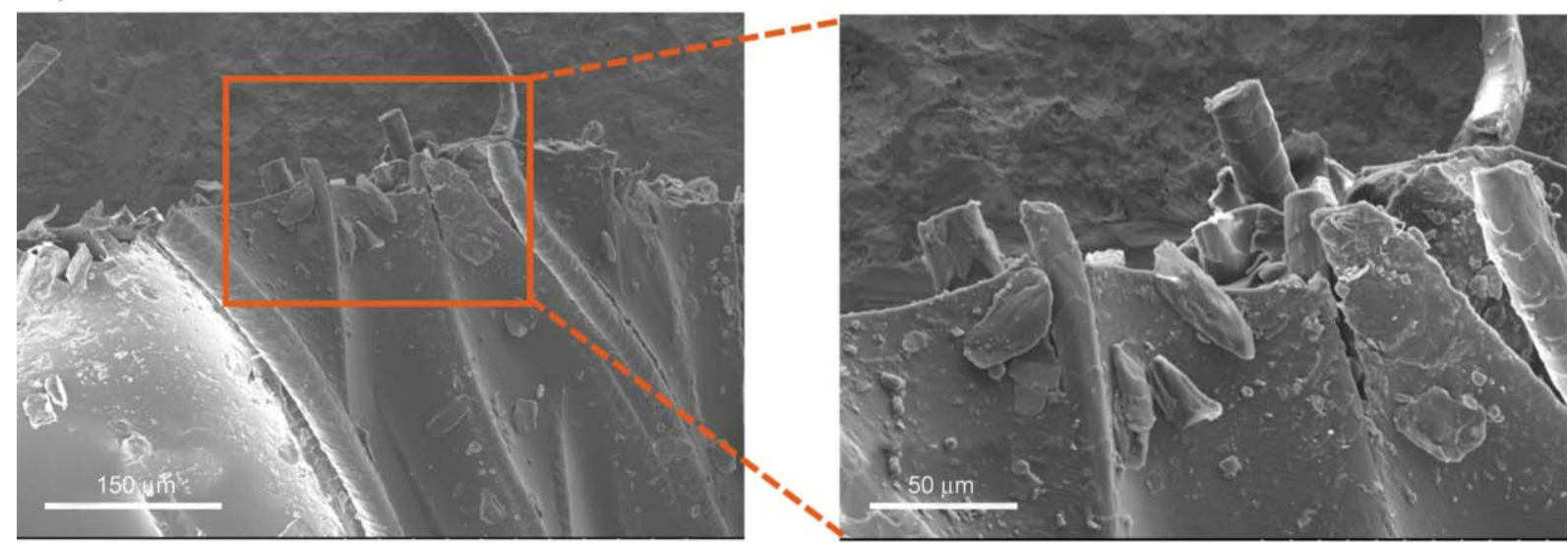

b)
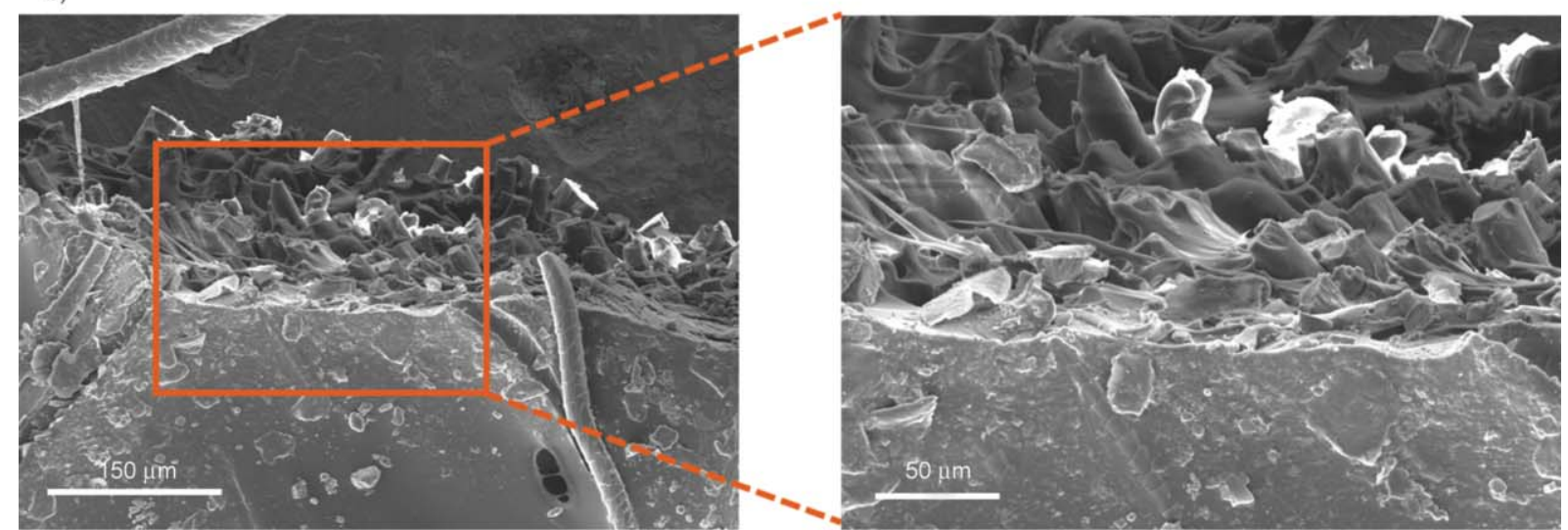

c)
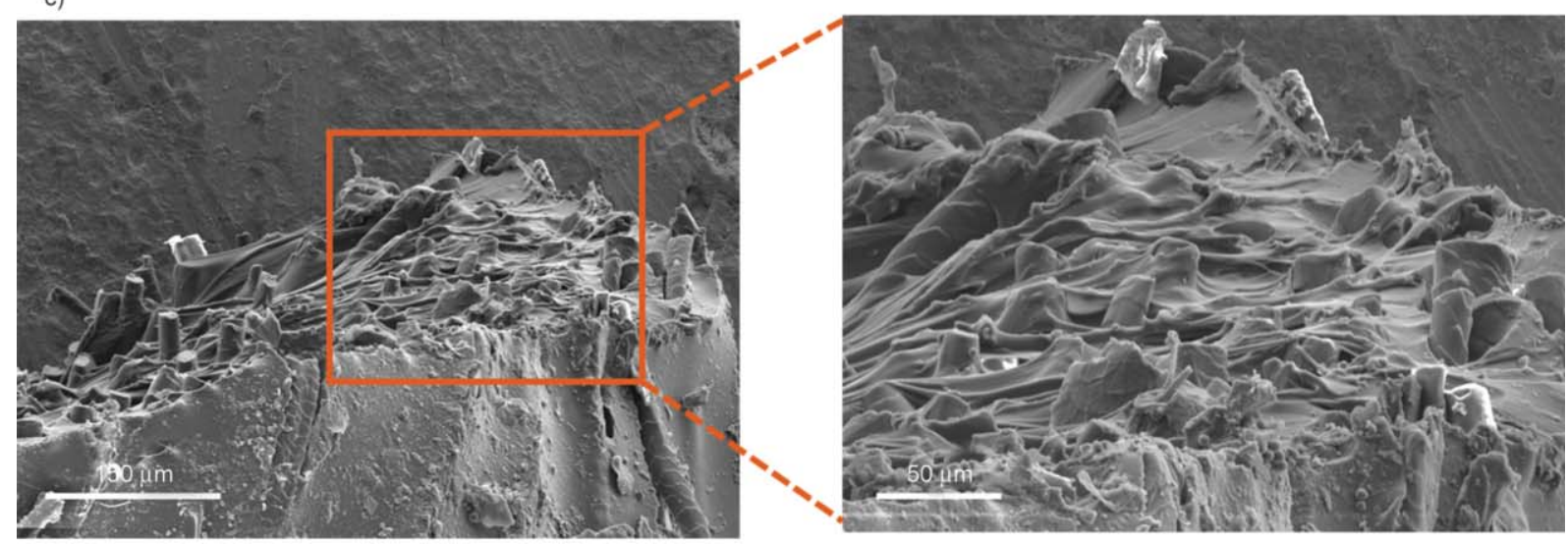

d)

Figure 6. SEM photos of the cross-sections of the wool yarns dip-coated with the pre-crosslinked PU solution for (a) once, (b) twice, (c) thrice, and (d) four times. 


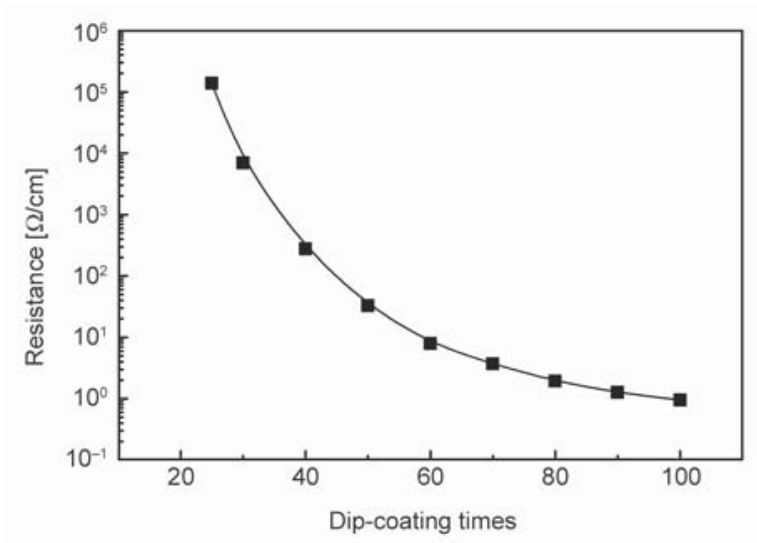

Figure 7. Electrical resistance per unit length of the PU@wool yarn dip-coated with the pre-crosslinked PU solution for two times as a function of the dip-coating times of AgNWs dispersion solution.

of the synthetic fibrous or fabric pressure sensors reported in the literature $[8,41-43]$ (Table 1).

On the other hand, the sensitivities of the sensors are inversely proportional to pressure, which approach to zero as pressure exceeds the threshold of about $\sim 3 \mathrm{kPa}$. Such a valid range is believed to be sufficient for portable applications like wearable electronics. Factually, the reduction in sensitivity to AgNWs loading and pressure is attributed to the same reason. The room for the AgNWs to move around as driven by the external stress greatly decreases when a higher concentration of AgNWs is added, or higher pressure is applied. That is, variations in AgNWs content and applied pressure are equivalent under the circumstances.

A more careful survey of Figure 10a reveals that the sensor made from the conductive yarns, which had been dip-coated with the pre-crosslinked PU solution for twice, has higher sensitivity than that from the yarns with thicker PU but the same AgNWs content (c.f. AgNWs(70)@PU(2)@wool and AgNWs(70)@PU(4)@wool).According to the negative correlation between the sensitivity and AgNWs content revealed in the last section, however, the sensitivity of the sensor derived from
AgNWs(70)@PU(4)@wool should be higher than that from AgNWs(70)@PU(2)@wool, as the relative amount of AgNWs in the former is smaller. Evidently, this is not the case. The SEM photos in Figure 5 demonstrate that the AgNWs(70)@PU(2)@wool yarn can keep the uneven structural characteristics of wool fibers, while the AgNWs(70)@PU(4)@wool yarn cannot. It implies that the contribution of the contact situations of the assembled yarns to the sensor sensitivities should be considered, which is more prominent than the amount of AgNWs. Having been stacked together, the conductive composite yarns with a smooth surface must have a larger contact area than those with irregular surface profile under the same force (Figure 10b). As a result, the stress would be spread out over larger regions in the former case, and the compression-induced deformation degree of conduction networks of $\mathrm{AgNWs}$, i.e. the responsivity, has to be smaller, leading to lower sensitivity accordingly.

Returning to the discussion about the conductive composite yarns, which concludes that the requirements of the yarn's stability and sensitivity for the AgNWs content are conflicting, we find the factors that can be united on the sensor. Because the surface profile of the conductive yarns is more critical for high sensitivity of the sensor, relatively less PU is enough so long as its content accords with the bottommost request (i.e. the dip-coating times of the pre-crosslinked $\mathrm{PU}=2$ ). It means that the one-sidedly pursuit of low AgNWs content of the conductive yarns is no longer necessary, and slightly higher AgNWs content is accepted for ensuring high sensitivity of the sensor and stability of the yarns as well. In this context, the sensor composed by the $\operatorname{AgNWs}(70) @ \mathrm{PU}(2) @ w o o l$ yarns is used for the rest studies in the following, which are more focused on application.

First, the reproducibility of the pressure sensing performance of the sensor is evaluated. As shown in Figure 11a, ten consecutive step pressures ranging

Table 1. Sensitivities of representative fibrous or fabric pressure sensors compared with those reported in this work.

\begin{tabular}{|c|c|c|c|}
\hline Sensor composition & Sensing mechanism & Sensitivity & References \\
\hline Ag nanoparticles/styrene-butadiene-styrene (SBS)@Kevlar fiber & Capacitive & $0.21 \mathrm{kPa}^{-1}$ & {$[8]$} \\
\hline Styrene-butadiene-styrene (SBS)/AgNWs@PU fiber & Piezoresistive & $0.20 \mathrm{kPa}^{-1}$ & {$[41]$} \\
\hline $\begin{array}{l}\text { Conductive fabric@microporous dielectric layer@conductive fabric (the fabric } \\
\text { was derived from Lycra elastomeric yarns) }\end{array}$ & Capacitive & $0.0121 \mathrm{kPa}^{-1}$ & {$[42]$} \\
\hline Aunano-popcorn-MoS $\mathrm{M}_{2} @$ polyester fiber & Piezoresistive & $0.19 \mathrm{kPa}^{-1}$ & {$[43]$} \\
\hline AgNWs(40)@PU(2)@wool yarn & Piezoresistive & $0.69 \mathrm{kPa}^{-1}$ & This work \\
\hline AgNWs(70)@PU(2)@wool yarn & Piezoresistive & $0.29 \mathrm{kPa}^{-1}$ & This work \\
\hline
\end{tabular}




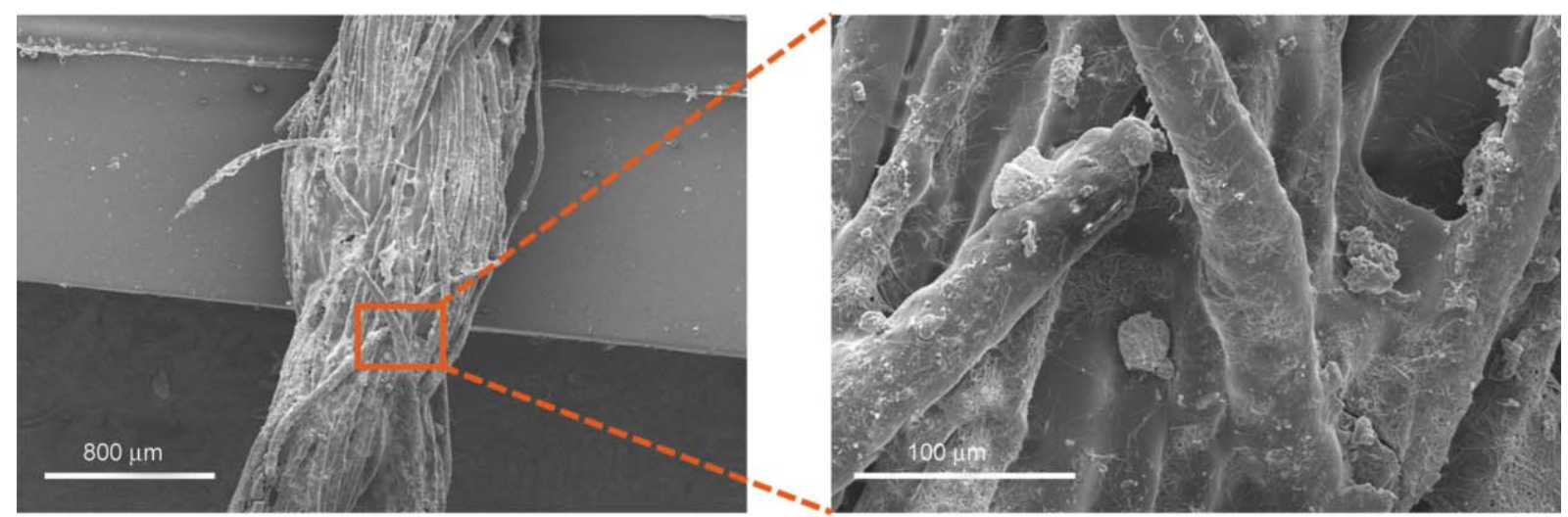

a)

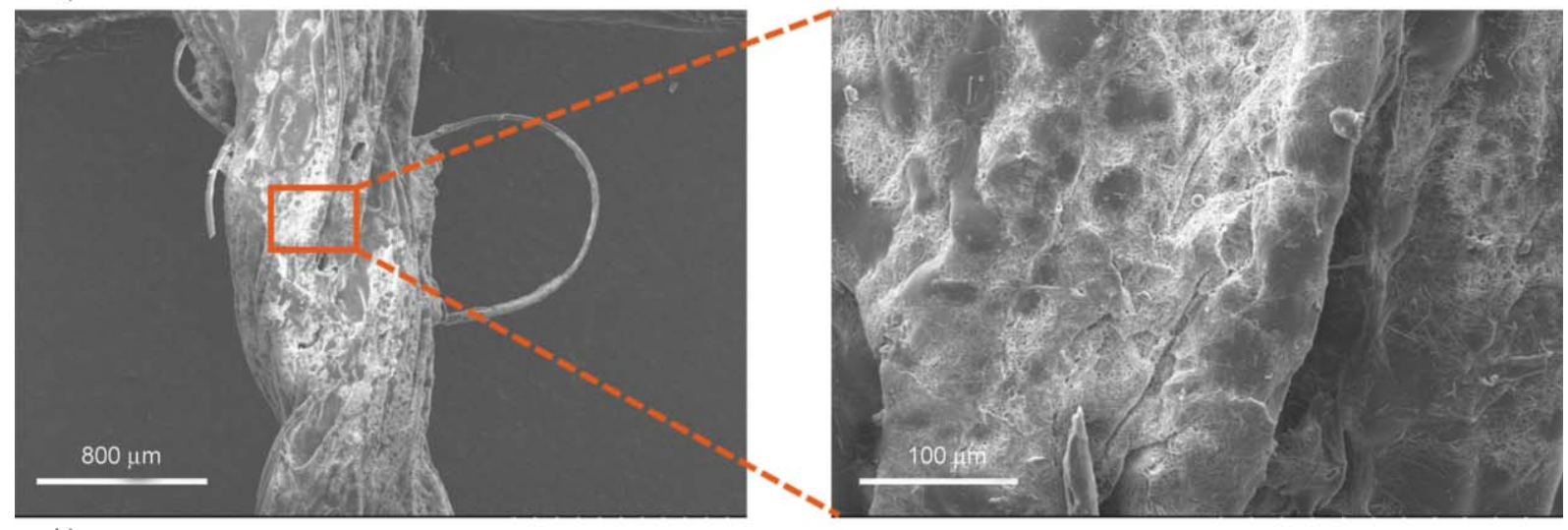

b)

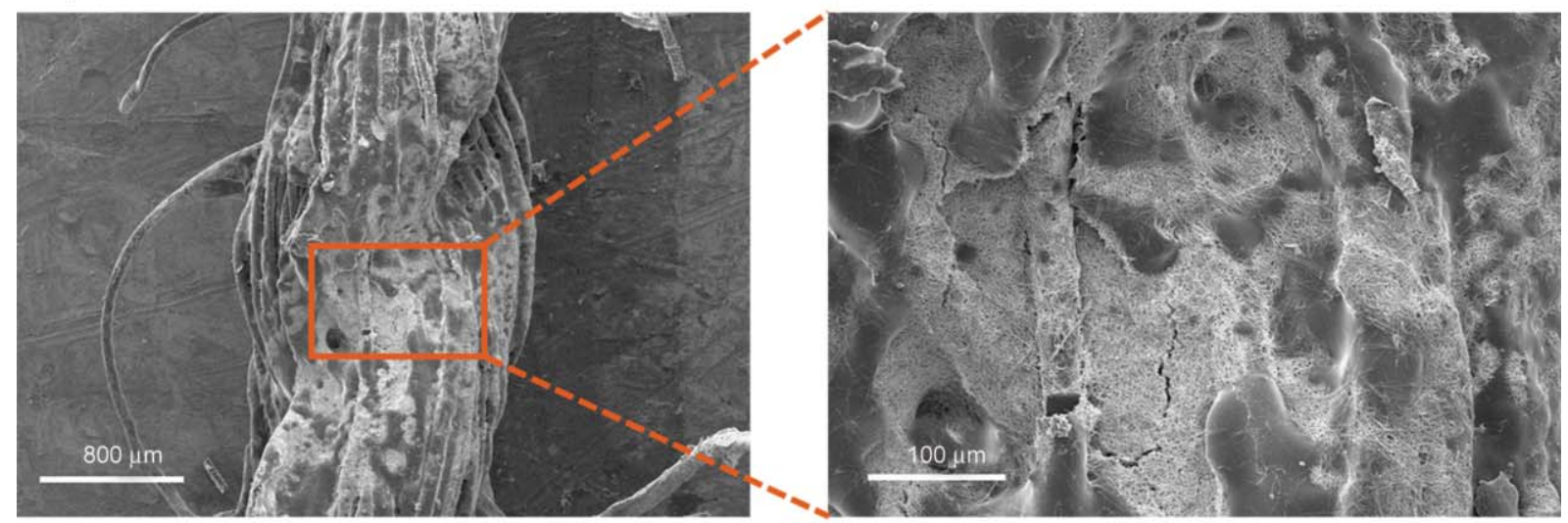

c)

Figure 8. SEM photos of (a) AgNWs(40)@PU(2)@wool, (b) AgNWs(70)@PU(2)@wool, and (c) AgNWs(100)@PU(2)@wool yarns.

from 0.2 to $2 \mathrm{kPa}$ were applied to the sensor, and the uniform signals in response to each pressure confirm the excellent reproducibility. Furthermore, ultralow step pressures are kept on being applied to collect the response signals in hopes of determining the detection limit (Figure 11b). It is found that when the pressure is reduced to $5 \mathrm{~Pa}$, the resistance variations are still accompanied by acceptable reproducibility. It manifests that the sensor is able to identify the pressure as low as $5 \mathrm{~Pa}$. The detection limit is among the best of fibrous sensor ever reported [44, 45]. Response time is another key parameter of sensors. The real-time resistance variation of the present sensor is recorded (Figure 11c) when a small glass plate is dropped onto the sensing pixel, which gets rid of the response time of the universal tester. Supposing the response time is defined as the time interval between 10 and $90 \%$ of the steady-state resistance [46], a response time of $28 \mathrm{~ms}$ is captured (Figure 11c), which is shorter than the value of human skin $(30-50 \mathrm{~ms})$ [47]. Lastly, a cyclic compression test is conducted to understand the durability of the sensor, which is always indispensable for practical and long-tern usages. As can be seen in Figure 11d, the resistance variation at a pressure of $0.2 \mathrm{kPa}$ shows stable responses within 5000 cycles. It is thus known that the composite 

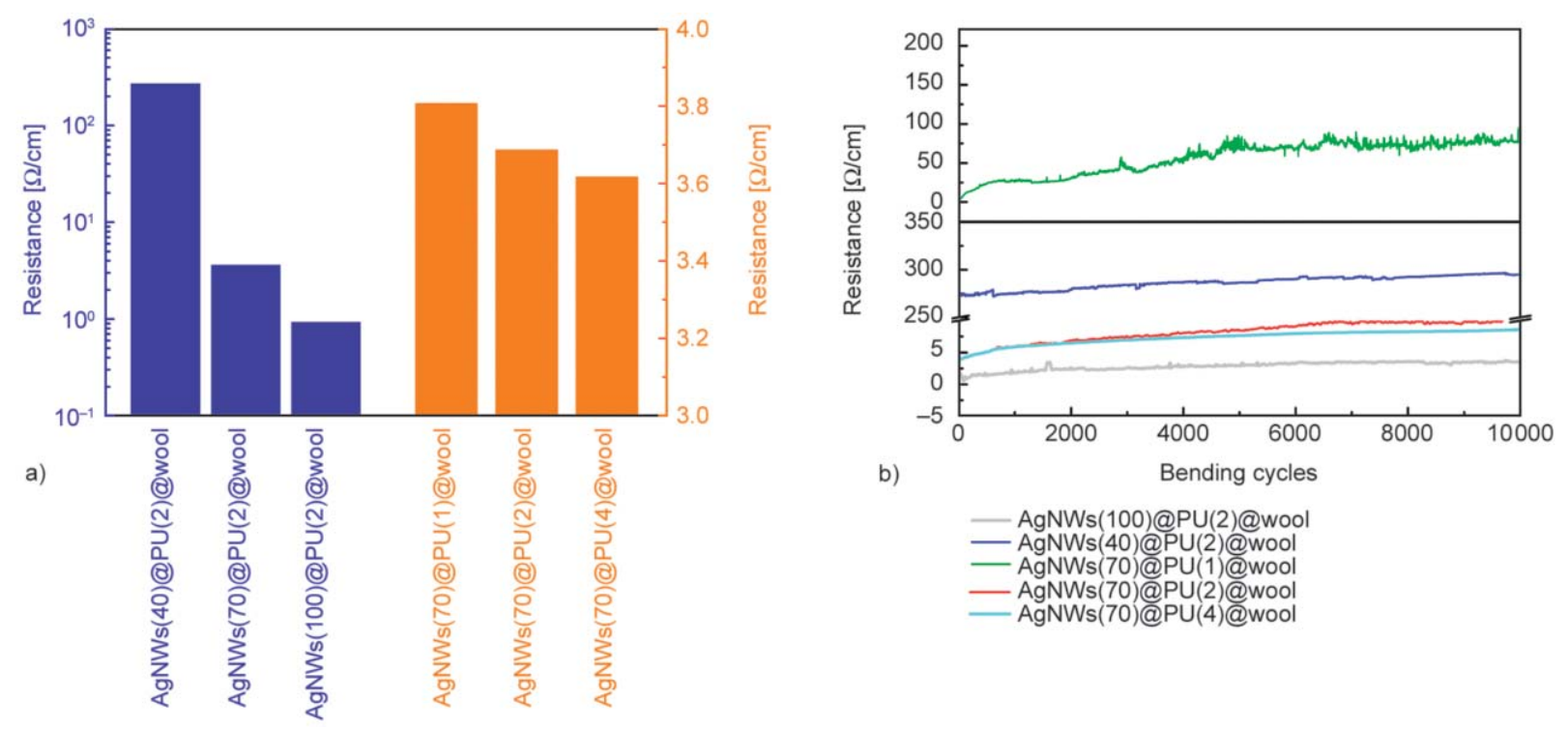

Figure 9. (a) Electrical resistance per unit length of AgNWs@PU@wool yarns as a function of the dip-coating times of AgNWs and pre-crosslinked PU solutions. (b) Dependences of electrical resistance per unit length of AgNWs@PU@wool yarns on bending cycles.

structure built up by AgNWs/PU on wool is quite steady under cyclic loading conditions.

It is noteworthy that the welded AgNWs networks have made an important contribution to the attractive performance exhibited in Figure 11. By taking advantage of plasma treatment, the assembly of the separated AgNWs turns into a compactly welded network [40] (Figure 3) so that it can deform as a whole under the applied pressure. In other words, the localized motion of individual AgNWs is replaced by the collective action of the networked AgNWs, which offers a more significant resistive response for detection.

Self-healing is an important measure to improve the durability of a material [48-50]. Even though microcracks are initiated, they can be eliminated in time through this interesting function without worrying about catastrophic failure. Our previous work [40] had developed the polyurethane used in the current sensor. It is able to self-heal damages in terms of the low concentration UV light in the sunlight that triggers disulfide metathesis assisted by the intermolecular hydrogen bonds. Because of the higher rapidity of hydrogen bonds formation than the reversible exchange of disulfide bonds, cracks can be firstly closed within a short time via hydrogen bonds and then covalently re-bonded by disulfide bonds. To check whether the self-healability is inherited by the conductive composite yarns, man-made microcracks are created and exposed to natural sunlight for restoration (refer to the Experimental for details). Figures 12a and $12 \mathrm{~b}$ demonstrate that the microcracks can indeed
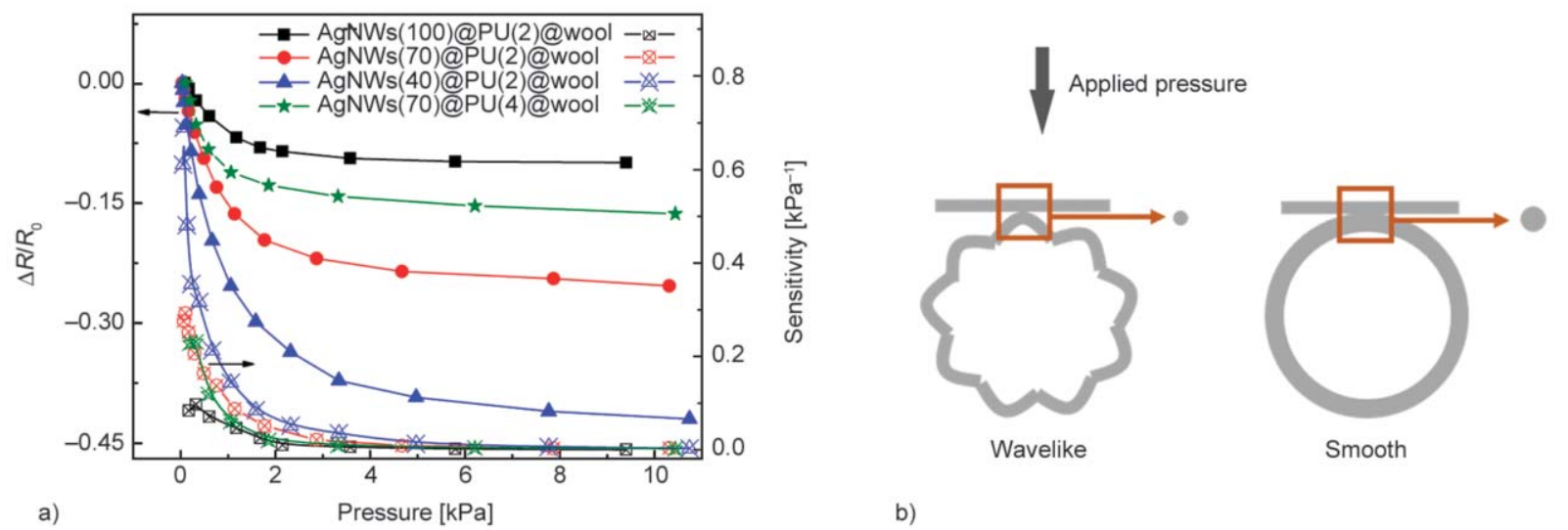

Figure 10. (a) Pressure dependences of relative resistance change and sensitivity of the pressure sensors assembled by different conductive composite yarns. (b) Illustrations of the contact areas of the sensing pixels composed of the orthogonally stacked conductive yarns with irregular and smooth surfaces under the applied pressure. 

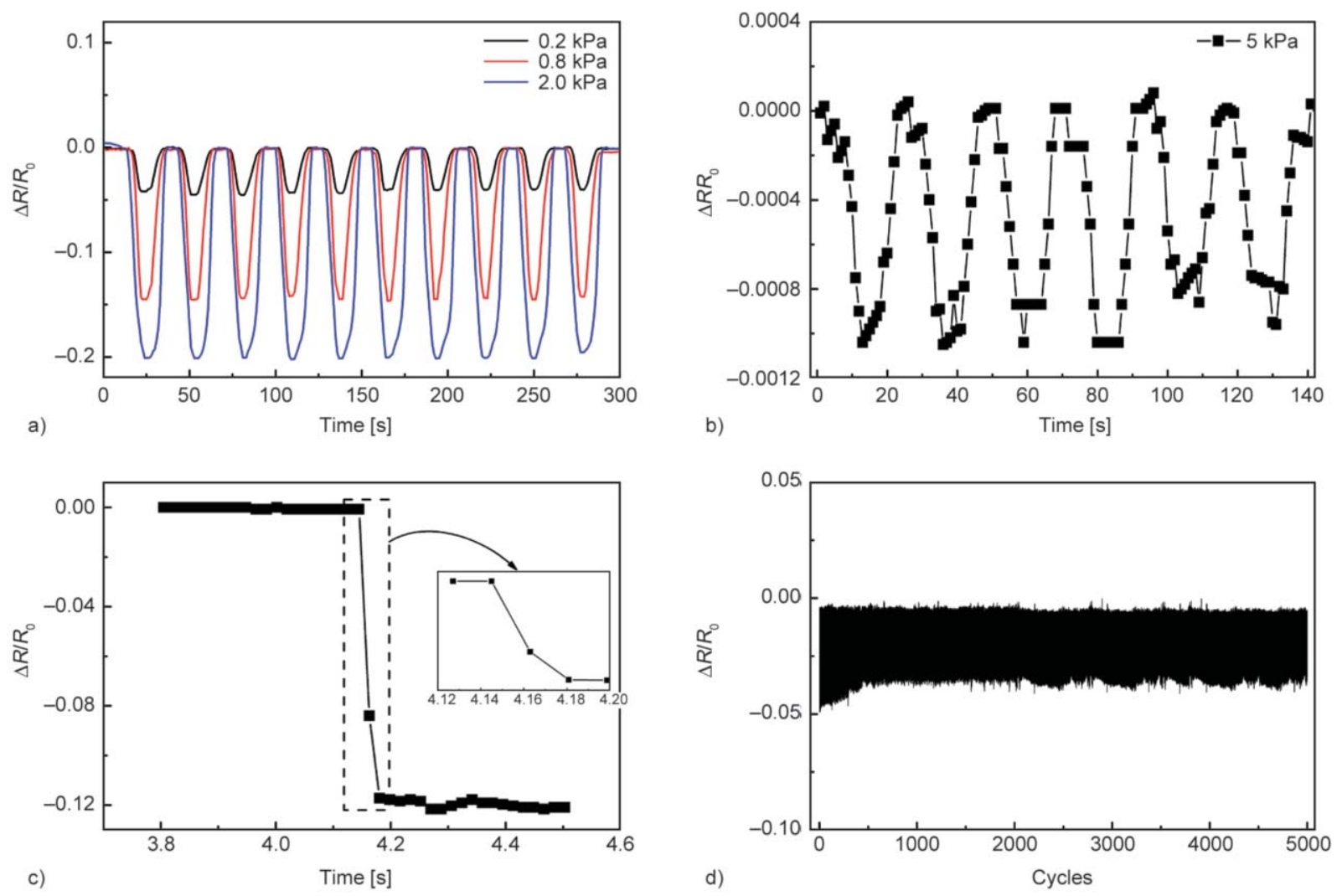

Figure 11. Time dependences of relative resistance variation of the pressure sensor measured (a) under different pressures from 0.2 to $2.0 \mathrm{kPa}$, (b) under an ultralow pressure of $5 \mathrm{~Pa}$, (c) when a small glass plate is dropped from a high place to the center of the sensing pixel, and (d) under constant pressure of $0.2 \mathrm{kPa}$ during 5000 compressive cycles. Note: The pressure sensor for these experiments is made from AgNWs(70)@PU(2)@wool yarns.

be repaired under the sun as expected. Meanwhile, the conductivity of the damaged conductive composite yarn increases with a rise in healing time (Figure 12c). In detail, the electrical resistance decreases slowly with healing time in the beginning and ending stages, but drops quickly in the medium term. An efficiency of about $98 \%$ of conductivity restoration is perceived. It proves that the composite yarn can be self-healed for recovering its electrical resistance by means of sunlight illumination because the separated AgNWs of the damaged conduction pathways are pulled together at the interface by the cracked PU matrix that is being healed.

Since AgNWs and PU have formed a composite, its self-healing performance should be affected by the component contents. As exhibited in Figure 12d, the electrical healing efficiencies of the yarns dip-coated with PU for two times and AgNWs solution for 40, 70 , and 100 times are 45.6, 98.0, and 98.8\%, respectively. The denser AgNWs networks suggest a higher re-connection probability of the broken conduction paths under the same circumstances, and hence higher electrical healing efficiency is achieved. Besides, in the case of the same AgNWs content, the fewer PU means less self-healable matrix to bring back the dislocated AgNWs. Consequently, the electrical healing efficiency of AgNWs(70)@PU(1)@wool is only $29.4 \%$, which is much lower than those of AgNWs(70)@PU(2)@wool (98.0\%) and AgNWs(70)@PU(4)@wool (99.2\%).

In addition to the static characterization of the electrical self-healing behavior of the conductive composite yarns, the dynamic responses of the pressure sensor assembled by the healed yarns are also detected. Figure 13a indicates that the healed sensor behaves like the original version. The healed portions of the composite yarns survive the cyclic compression. It implies that the tiny damages of the sensor are allowed to be unconsciously repaired when enjoying outdoor activities. For reference, the damaged specimen was placed in the dark for $24 \mathrm{~h}$ and then tested under the same conditions. Because the PU can not be self-healed in the absence of sunlight, cracks propagation and coalescence have to be promoted under the repeated loading-unloading. The resistance of the specimen keeps on increasing as a result (Figure 13b). Eventually, 


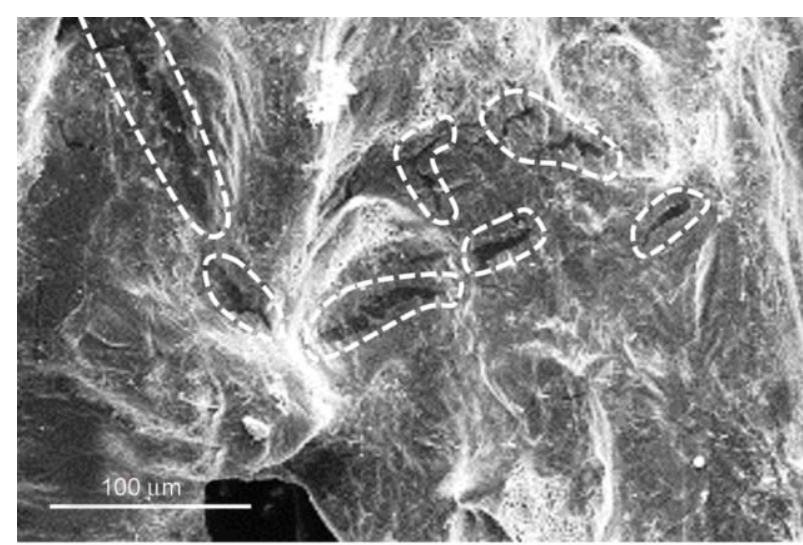

a)

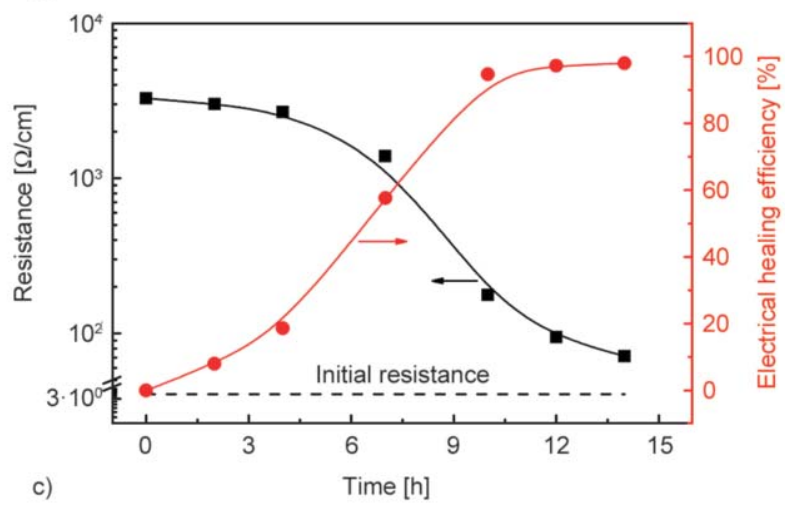

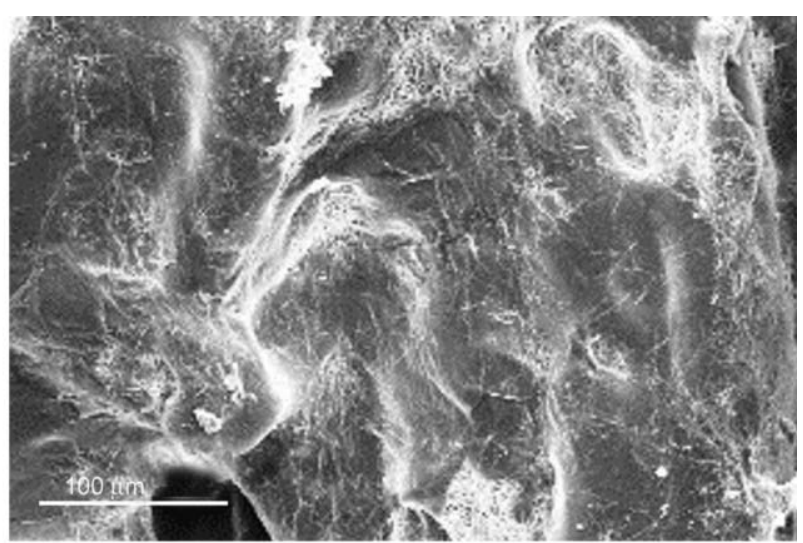

b)

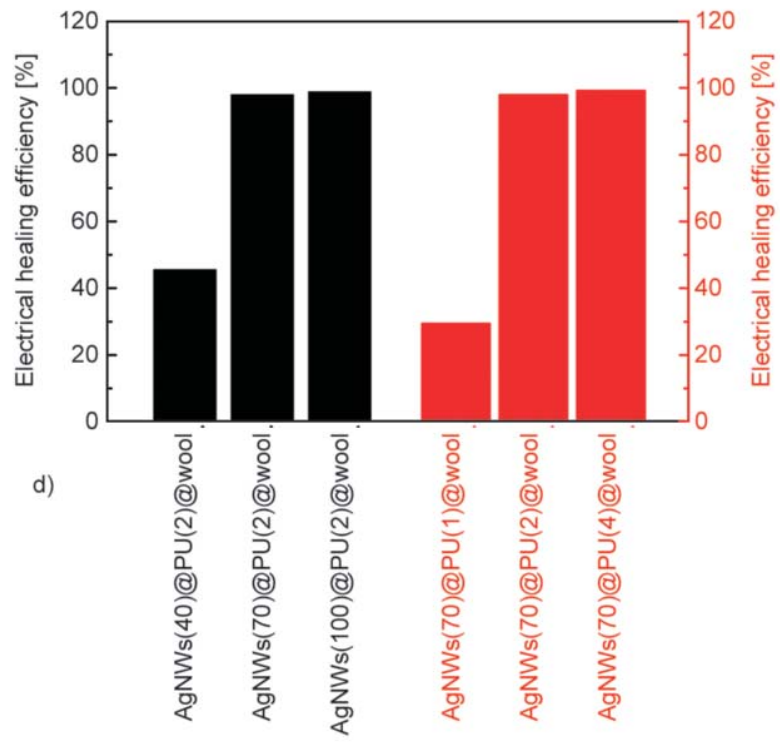

Figure 12. SEM images of (a) the cracked (circled by while dash lines) and (b) the sunlight healed surface of AgNWs(70)@PU(2)@wool yarns. (c) Resistance per unit length and electrical healing efficiency of AgNWs(70)@PU(2)@wool yarn as a function of sunlight healing time. (d) Effect of the dip-coating times of AgNWs and pre-crosslinked PU solutions on the electrical healing efficiency of AgNWs@PU@wool yarns.

unstable propagation of cracks happens, and the specimen fails only after 357 cycles. The service life of

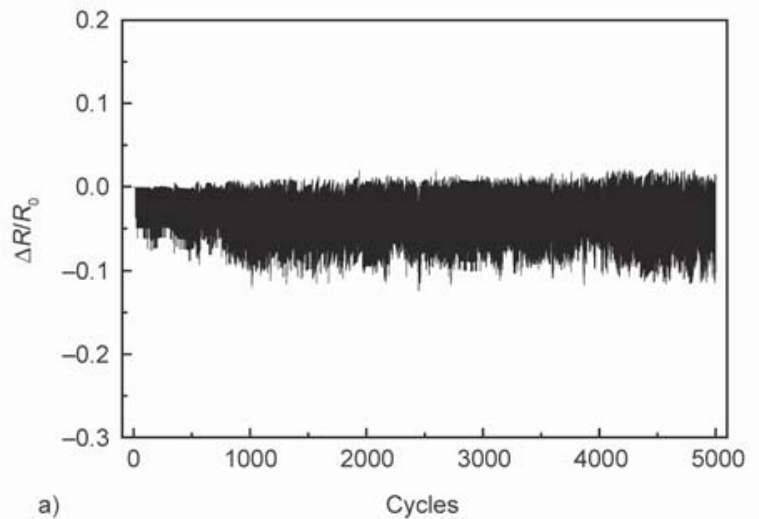

the sensor is much shorter than those of the original (Figure 11d) and healed versions (Figure 13a).

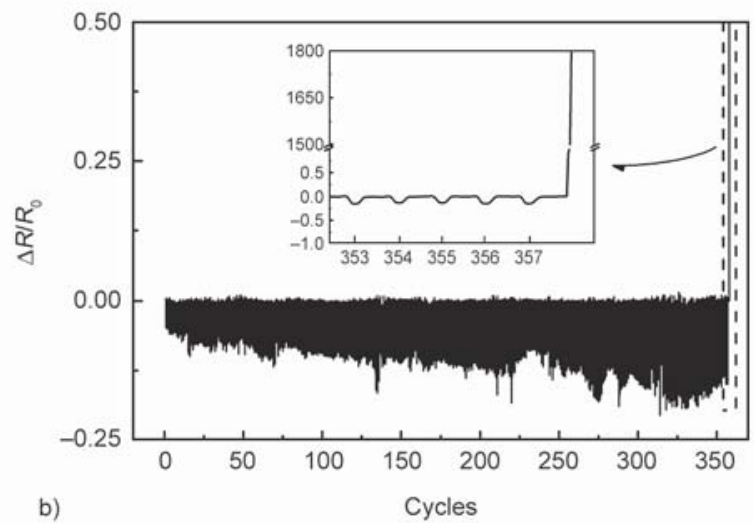

Figure 13. (a) Relative resistance changes of the sensor assembled by the AgNWs(70)@PU(2)@wool yarns (that had been damaged and healed with sunlight) measured under repeated compression. (b) Relative resistance changes of the sensor assembled by the AgNWs(70)@PU(2)@wool yarns (that had been damaged and placed in dark for 24 h) measured under repeated compression. Note: The maximum applied pressure $=0.2 \mathrm{kPa}$. 
In the last part of this paper, the performance of the pressure sensor in practical scenarios is assessed. To this end, the sensors tightly adhere to the wrist joint, human throat, the radial artery on the wrist, and pocket radio speaker to monitor human motion, vocalization, pulse beat, and sound vibration as a function of time, respectively. As exhibited in Figure 14a, when the wrist bends, the pressure aroused by the movement is immediately applied to the sensing pixel, and the resistance decreases accordingly. When a male adult spoke, the resistance variations caused by the throat vibration can distinguish the trisyllabic word 'polymer'(refer to the three small peaks in the
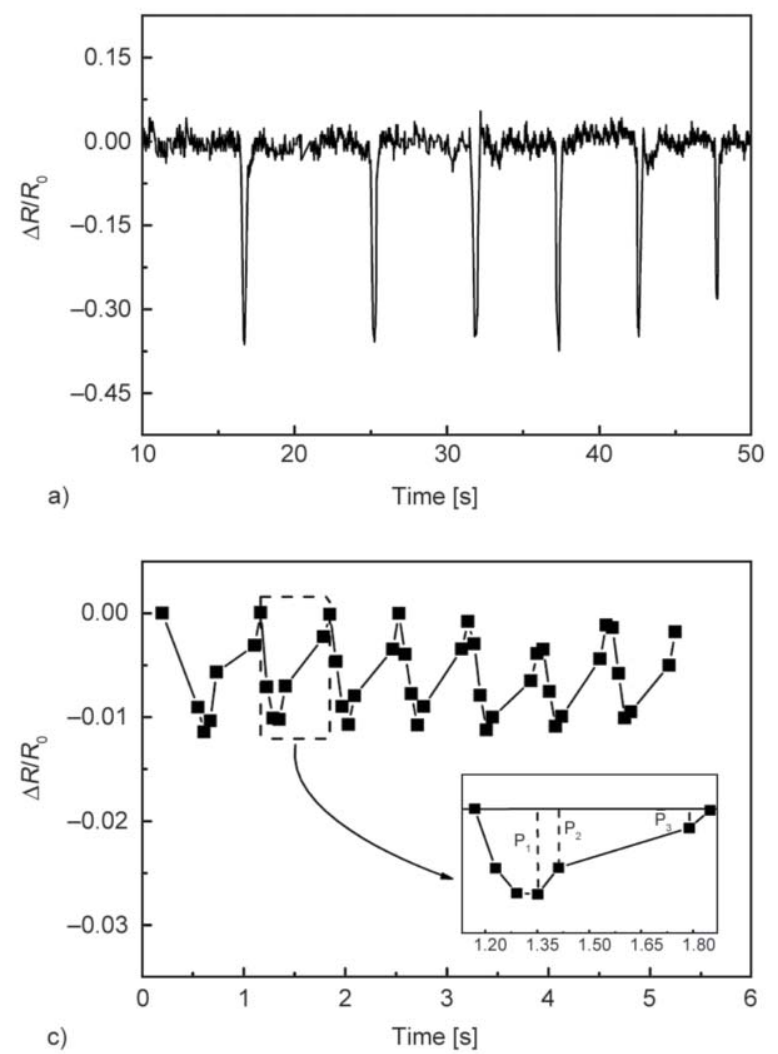

inset of Figure 14b), owing to the fast and elaborate responsive features of the sensor. Unlike the higher pressures generated by wrist bending or throat movement, pulse beat and vibration of radio speakers are much weaker. Figure 14c shows the signals of our sensor in response to radial artery pressure waves. Every cycle is composed of three component waves: the incident wave from heartbeat, reflective wave from hand, and reflective wave from the lower part of the body [51]. The data can be used to determine the radial augmentation index, AIr, defined as P2/P1 in the enlarged inset of Figure 14c, which is an indicator related to arterial stiffness [52]. Accordingly,
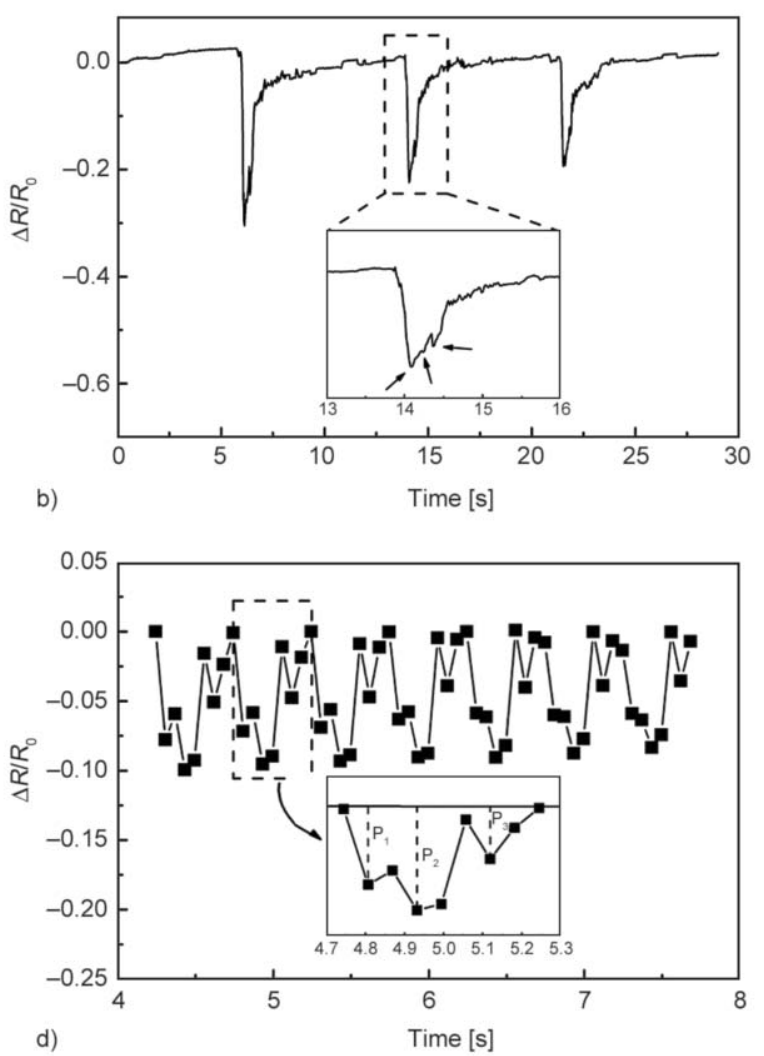

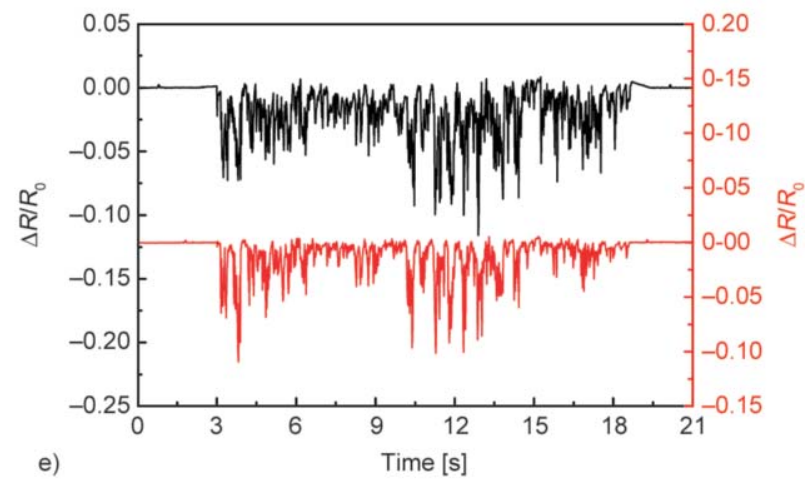

Figure 14. Time dependences of relative resistance variations of the pressure sensor assembled by the AgNWs(70)@PU(2)@wool yarns in response to (a) wrist bending, (b) throat vibration when speaking 'polymer,' (c) pulse beat of a male adult, (d) pulse beat of a female hypotensive adult, and (e) sound-driven vibration from a pocket radio speaker when playing music. 
the AIr value of the volunteer ( 28 years old man) is calculated to be 0.69 from Figure 14c, which is typical for people in the same age range. In addition, the pulse beats of a female hypotensive adult are also recorded by the pressure sensor assembled by the AgNWs(70)@PU(2)@wool yarns (Figure 14d).Her AIr is estimated to be 1.33, outside of the normal range (Air < 1). The results demonstrate the usefulness of our pressure sensor for the primary diagnosis of the diseases related to pulse beats. In general, the AIr data are measured by commercial blood pressure monitor. The present work provides a much simpler substitute prototype. As for the pressure sensor attached to the speaker of a pocket radio, the resistance can successfully keep pace with the sound vibration (Figure 14e) when the pop music 'poker face' is played. The signals are clearly distinguished following the changes in volume and rhythm and repetitive well for two tests.

\section{Conclusions}

A self-healable, electrically conductive, and flexible wool-based resistive-type pressure sensor is successfully developed, which possesses high sensitivity, low detection limit, short response time, and outstanding working stability. It proves to be able to distinguish signals with different strengths and frequencies from real-life scenarios. The rational design of materials formulation and structure plays a critical role. In particular, the pliability and surface irregularity of wool, the balanced properties and self-healability of the PU, the high electro-conductivity and robustness of the AgNWs networks, the loadings of PU and AgNWs, and the junction status of the conductive composite yarns are the key factors responsible for the high performance of the sensor.

Owing to the nature of wool, the present fibrous pressure sensor can be easily combined with conventional textiles or woven into 2D or 3D forms or specialshaped structures, which will provide new opportunities for diverse applications in the future.

\section{Acknowledgements}

The authors thank the support of the National Natural Science Foundation of China (Grants: 51773229, 51333008, 51673219 and 51873235), the Scientific and Technological Program of Guangdong Province (Grant: 2017A010103008), and the Science Foundation of Guangdong Province (Grant: 2018B030311017).

\section{References}

[1] Wang Z., Si Y., Zhao C., Yu D., Wang W., Sun G.: Flexible and washable poly(ionic liquid) nanofibrous membrane with moisture proof pressure sensing for real-life wearable electronics. ACS Applied Materials and Interfaces, 11, 27200-27209 (2019). https://doi.org/10.1021/acsami.9b07786

[2] Li Y., Samad Y. A., Liao K.: From cotton to wearable pressure sensor. Journal of Materials Chemistry A, 3, 2181-2187 (2015). https://doi.org/10.1039/C4TA05810K

[3] Wan C., Chen G., Fu Y., Wang M., Matsuhisa N., Pan S., Pan L., Yang H., Wan Q., Zhu L., Chen X.: An artificial sensory neuron with tactile perceptual learning. Advanced Materials, 30, 1801291/1-1801291/7 (2018). https://doi.org/10.1002/adma.201801291

[4] Wang M., Wang W., Leow W. R., Wan C., Chen G., Zeng Y., Yu J., Liu Y., Cai P., Wang H., Lelmini D., Chen X.: Enhancing the matrix addressing of flexible sensory arrays by a highly nonlinear threshold switch. Advanced Materials, 30, 1802516/1-1802516/9 (2018). https://doi.org/10.1002/adma.201802516

[5] Gao Y., Ota H., Schaler E. W., Chen K., Zhao A., Gao W., Fahad H. M., Leng Y., Zheng A., Xiong F., Zhang C., Tai L-C., Zhao P., Fearing R. S., Javey A.: Wearable microfluidic diaphragm pressure sensor for health and tactile touch monitoring. Advanced Materials, 29, 1701985/1-1701985/8 (2017). https://doi.org/10.1002/adma.201701985

[6] Gong S., Schwalb W., Wang Y., Chen Y., Tang Y., Si J., Shirinzadeh B., Cheng W.: A wearable and highly sensitive pressure sensor with ultrathin gold nanowires. Nature Communications, 5, 3132/1-3132/8 (2014). https://doi.org/10.1038/ncomms4132

[7] Guo Y., Zhong M., Fang Z., Wan P., Yu G.: A wearable transient pressure sensor made with mxene nanosheets for sensitive broad-range human-machine interfacing. Nano Letters, 19, 1143-1150 (2019).

https://doi.org/10.1021/acs.nanolett.8b04514

[8] Lee J., Kwon H., Seo J., Shin S., Koo J. H., Pang C., Son S., Kim J. H., Jang Y. H., Kim D. E., Lee T.: Conductive fiber-based ultrasensitive textile pressure sensor for wearable electronics. Advanced Materials, 27, 2433 2439 (2015).

https://doi.org/10.1002/adma.201500009

[9] Kang B-C., Ha T-J.: Human-interactive drone system remotely controlled by printed strain/pressure sensors consisting of carbon-based nanocomposites. Composite Science and Technology, 182, 107784/1-107784/9 (2019).

https://doi.org/10.1016/j.compscitech.2019.107784

[10] Hua Q., Sun J., Liu H., Bao R., Yu R., Zhai J., Pan C., Wang Z. L.: Skin-inspired highly stretchable and conformable matrix networks for multifunctional sensing. Nature Communications, 9, 244/1-244/11 (2018). https://doi.org/10.1038/s41467-017-02685-9 
[11] Yu L., Yeo J. C., Soon R. H., Yeo T., Lee H. H., Lim C. T.: Highly stretchable, weavable, and washable piezoresistive microfiber sensors. ACS Applied Materials and Interfaces, 10, 12773-12780 (2018). https://doi.org/10.1021/acsami.7b19823

[12] Jeong H., Noh Y., Ko S. H., Lee D.: Flexible resistive pressure sensor with silver nanowire networks embedded in polymer using natural formation of air gap. Composite Science and Technology, 174, 50-57 (2019). https://doi.org/10.1016/j.compscitech.2019.01.028

[13] Chen Z., Wang Z., Li X., Lin Y., Luo N., Long M., Zhao N., Xu J-B.: Flexible piezoelectric-induced pressure sensors for static measurements based on nanowires/ graphene heterostructures. ACS Nano, 11, 4507-4513 (2017). https://doi.org/10.1021/acsnano.6b08027

[14] Tien N. T., Jeon S., Kim D-I., Trung T. Q., Jang M., Hwang B-U., Byun K-E., Bae J., Lee E., Tok J. B-H., Bao Z., Lee N-E., Park J-J.: A flexible bimodal sensor array for simultaneous sensing of pressure and temperature. Advanced Materials, 26, 796-804 (2014). https://doi.org/10.1002/adma.201302869

[15] Takamtsu S., Lonjaret T., Ismailova E., Masuda A., Itoh T., Malliaras G. G.: Wearable keyboard using conducting polymer electrodes on textiles. Advanced Materials, 28, 4485-4488 (2016). https://doi.org/10.1002/adma.201504249

[16] Li R., Si Y., Zhu Z., Guo Y., Zhang Y., Pan N., Sun G., Pan T.: Supercapacitive iontronic nanofabric sensing. Advanced Materials, 29, 1700253/1-1700253/8 (2017). https://doi.org/10.1002/adma.201700253

[17] Lin Z., Chen J., Li X., Zhou Z., Meng K., Wei W., Yang J., Wang Z. L.: Triboelectric nanogenerator enabled body sensor network for self-powered human heart-rate monitoring. ACS Nano, 11, 8830-8837 (2017). https://doi.org/10.1021/acsnano.7b02975

[18] Dhakar L., Gudla S., Shan X., Wang Z., Tay F. E. H., Heng C-H., Lee C.: Large scale triboelectric nanogenerator and self-powered pressure sensor array using low cost roll-to-roll UV embossing. Scientific Reports, 6, 22253/1-22253/10 (2016).

https://doi.org/10.1038/srep22253

[19] Fuh Y-K., Li S-C., Chen C-Y., Tsai C-Y.: A fully packaged self-powered sensor based on near-field electrospun arrays of poly(vinylidene fluoride) nano/micro fibers. Express Polymer Letters, 12, 136-145 (2018). https://doi.org/10.3144/expresspolymlett.2018.12

[20] Tang X., Wu C., Gan L., Zhang T., Zhou T., Huang J., Wang H., Xie C., Zeng D.: Multilevel microstructured flexible pressure sensors with ultrahigh sensitivity and ultrawide pressure range for versatile electronic skins. Small, 15, 1804559/1-1804559/9 (2019). https://doi.org/10.1002/smll.201804559

[21] He J., Xiao P., Lu W., Shi J., Zhang L., Liang Y., Pan C., Kuo S-W., Chen T.: A universal high accuracy wearable pulse monitoring system via high sensitivity and large linearity graphene pressure sensor. Nano Energy, 59, 422-433 (2019).

https://doi.org/10.1016/j.nanoen.2019.02.036
[22] Zhou X., Zhang Y., Yang J., Li J., Luo S., Wei D.: Flexible and highly sensitive pressure sensors based on microstructured carbon nanowalls electrodes. Nanomaterials, 9, 496/1-496/11 (2019). https://doi.org/10.3390/nano9040496

[23] Xu J., Pei L., Li J., Pang H., Li Z., Li B., Xuan S., Gong X.: Flexible, self-powered, magnetism/pressure dualmode sensor based on magnetorheological plastomer. Composite Science and Technology, 183, 107820/1107820/9 (2019).

https://doi.org/10.1016/j.compscitech.2019.107820

[24] Krawczak P.: Polymer-based sensors: A high potential to be further developed. Express Polymer Letters, 3, 400 (2009).

https://doi.org/10.3144/expresspolymlett.2009.49

[25] Zhou J., Tian G., Jin G., Xin Y., Tao R., Lubineau G.: Buckled conductive polymer ribbons in elastomer channels as stretchable fiber conductor. Advanced Functional Materials, 30, 1907316/1-1907316/9 (2020). https://doi.org/10.1002/adfm.201907316

[26] Chen J., Li H., Yu Q., Hu Y., Cui X., Zhu Y., Jiang W.: Strain sensing behaviors of stretchable conductive polymer composites loaded with different dimensional conductive fillers. Composite Science and Technology, 168, 388-396 (2018). https://doi.org/10.1016/j.compscitech.2018.10.025

[27] Lee S., Shin S., Lee S., Seo J., Lee J., Son S., Cho H. J., Algadi H., Al-Sayari S., Kim D. E., Lee T.: Ag nanowire reinforced highly stretchable conductive fibers for wearable electronics. Advanced Functional Materials, 25, 3114-3121 (2015). https://doi.org/10.1002/adfm.201500628

[28] Cheng Y., Wang R., Sun J., Gao L.: Highly conductive and ultrastretchable electric circuits from covered yarns and silver nanowires. ACS Nano, 9, 3887-3895 (2015). https://doi.org/10.1021/nn5070937

[29] Ma R., Lee J., Choi D., Moon H., Baik S.: Knitted fabrics made from highly conductive stretchable fibers. Nano Letters, 14, 1944-1951 (2014).

https://doi.org/10.1021/nl404801t

[30] Liu Z. F., Fang S., Moura F. A., Ding J. N., Jiang N., Di J., Zhang M., Lepró X., Galvão D. S., Haines C. S., Yuan N. Y., Yin S. G., Lee D. W., Wang R., Wang H. Y., Lv W., Dong C., Zhang R. C., Chen M. J., Yin Q., Chong Y. T., Zhang R., Wang X., Lima M. D., OvalleRobles R., Qian D., Lu H., Baughman R. H.: Hierarchically buckled sheath-core fibers for superelastic electronics, sensors, and muscles. Science, 349, 400-404 (2015). https://doi.org/10.1126/science.aaa79522

[31] Ryu S., Lee P., Chou J. B., Xu R., Zhao R., Hart A. J., Kim S-G.: Extremely elastic wearable carbon nanotube fiber strain sensor for monitoring of human motion. ACS Nano, 9, 5929-5936 (2015). https://doi.org/10.1021/acsnano.5b00599 
[32] Eom J., Lee Y. R., Lee J. H., Park S. K., Jeong Y., Park J. S., Kim Y. H.: Highly conductive and stretchable fiber interconnections using dry-spun carbon nanotube fibers modified with ionic liquid/poly(vinylidene fluoride) copolymer composite. Composite Science and Technology, 169, 1-6 (2019).

https://doi.org/10.1016/j.compscitech.2018.10.035

[33] Xu Z., Zhang Y., Li P., Gao C.: Strong, conductive, lightweight, neat graphene aerogel fibers with aligned pores. ACS Nano, 6, 7103-7113 (2012).

https://doi.org/10.1021/nn3021772

[34] Cheng Y., Wang R., Sun J., Gao L.: A stretchable and highly sensitive graphene-based fiber for sensing tensile strain, bending, and torsion. Advanced Materials, 27, 7365-7371 (2015). https://doi.org/10.1002/adma.201503558

[35] Pan L., Chortos A., Yu G., Wang Y., Isaacson S., Allen R., Shi Y., Dauskardt R., Bao Z.: An ultra-sensitive resistive pressure sensor based on hollow-sphere microstructure induced elasticity in conducting polymer film. Nature Communications, 5, 3002/1-3002/8 (2014). https://doi.org/10.1038/ncomms4002

[36] Bae G. Y., Pak S. W., Kim D., Lee G., Kim D. H., Chung Y., Cho K.: Linearly and highly pressure-sensitive electronic skin based on a bioinspired hierarchical structural array. Advanced Materials, 28, 5300-5306 (2016). https://doi.org/10.1002/adma.201600408

[37] Kim K-H., Hong S-K., Jang N-S., Ha S-H., Lee H-W., Kim J-M.: Wearable resistive pressure sensor based on highly flexible carbon composite conductors with irregular surface morphology. ACS Applied Materials and Interfaces, 9, 17499-17507 (2017). https://doi.org/10.1021/acsami.7b06119

[38] Xu W. M., Rong M. Z., Zhang M. Q.: Sunlight driven self-healing, reshaping and recycling of a robust, transparent and yellowing-resistant polymer. Journal of Materials Chemistry A, 4, 10683-10690 (2016). https://doi.org/10.1039/C6TA02662A

[39] Song Y. X., Rong M. Z., Zhang M. Q.: Improvement of multiple-responsive shape memory effects of wool through increasing the content of disulfide bonds. Polymer, 188, 122130/1-122130/9 (2020). https://doi.org/10.1016/j.polymer.2019.122130

[40] Song Y. X., Xu W. M., Rong M. Z., Zhang M. Q.: A sunlight self-healable transparent strain sensor with high sensitivity and durability based on a silver nanowire/ polyurethane composite film. Journal of Materials Chemistry A, 7, 2315-2325 (2019).

https://doi.org/10.1039/C8TA11435H

[41] Chen S., Liu S., Wang P., Liu H., Liu L.: Highly stretchable fiber-shaped e-textiles for strain/pressure sensing, full-range human motions detection, health monitoring, and 2D force mapping. Journal of Materials Science, 53, 2995-3005 (2018). https://doi.org/10.1007/s10853-017-1644-y
[42] Atalay O., Atalay A., Gafford J., Walsh C.: A highly sensitive capacitive-based soft pressure sensor based on a conductive fabric and a microporous dielectric layer. Advanced Materials Technologies, 3, 1700237/11700237/8 (2017). https://doi.org/10.1002/admt.201700237

[43] Lan L., Zhao F., Yao Y., Ping J., Ying Y.: One-step and spontaneous in situ growth of popcorn-like nanostructures on stretchable double-twisted fiber for ultrasensitive textile pressure sensor. ACS Applied Materials and Interfaces, 12, 10689-10696 (2020).

https://doi.org/10.1021/acsami.0c00079

[44] Kim S. Y., Jee E., Kim J. S., Kim D. H.: Conformable and ionic textiles using sheath-core carbon nanotube microyarns for highly sensitive and reliable pressure sensors. RSC Advances, 7, 23820-23826 (2017). https://doi.org/10.1039/C7RA02215H

[45] Chhetry A., Yoon H., Park J. Y.: A flexible and highly sensitive capacitive pressure sensor based on conductive fibers with a microporous dielectric for wearable electronics. Journal of Materials Chemistry C, 5, 1006810076 (2017). https://doi.org/10.1039/C7TC02926H

[46] Yao S., Zhu Y.: Wearable multifunctional sensors using printed stretchable conductors made of silver nanowires. Nanoscale, 6, 2345-2352 (2014). https://doi.org/10.1039/C3NR05496A

[47] Wu W., Wen X., Wang Z. L.: Taxel-addressable matrix of vertical-nanowire piezotronic transistors for active and adaptive tactile imaging. Science, 340, 952-957 (2013). https://doi.org/10.1126/science.1234855

[48] Zhao T., Ai J., Wang P., Tong W., Ding C., Cen Y., Zhang M.: Research of the influence factors on transesterification reaction degree in PC/PBT blends. Advanced Industrial and Engineering Polymer Research, 2, 203-208 (2019). https://doi.org/10.1016/j.aiepr.2019.09.005

[49] Chen X-X., Zhong Q-Y., Wang S-J., Wu Y-S., Tan J-D., Lei H-X., Huang S-Y., Zhang Y-F.: Progress in dynamic covalent polymers (in Chinese). Acta Polymerica Sinica, 50, 469-484 (2019). https://doi.org/10.11777/j.issn1000-3304.2019.18277

[50] Zhang Z-P., Rong M-Z., Zhang M-Q.: Research progress of processing of crosslinked polymers based on reversible covalent chemistry: A new challenge to the development of polymer engineering (in Chinese). Acta Polymerica Sinica, 7, 829-853 (2018). https://doi.org/10.11777/j.issn1000-3304.2018.18060

[51] Nichols W. W.: Clinical measurement of arterial stiffness obtained from noninvasive pressure waveforms. American Journal of Hypertension, 18, 3S-10S (2005). https://doi.org/10.1016/j.amjhyper.2004.10.009

[52] Kohara K., Tabara Y., Oshiumi A., Miyawaki Y., Kobayashi T., Miki T.: Radial augmentation index: A useful and easily obtainable parameter for vascular aging. American Journal of Hypertension, 18, 11S-14S (2005).

https://doi.org/10.1016/j.amjhyper.2004.10.010 